DESY 94-188

\title{
Domain Wall Fermions and Chiral Gauge Theories
}

\author{
Karl Jansen \\ Deutsches Elektronen-Synchrotron DESY, \\ Notkestr. 85, D-22603 Hamburg, Germany
}

October, 1994

\begin{abstract}
We review the status of the domain wall fermion approach to construct chiral gauge theories on the lattice.
\end{abstract}

\section{Introduction}

It is a natural question to ask, whether there exists a regularization of a chiral gauge theory beyond perturbation theory. This addresses the pure existence of the Standard Model of electroweak interactions. The astonishing answer to the above question is that a non-perturbative regularization has so far not been found. Even worse, nogo theorems [1, 2] seem to make it impossible to even find such a regularization. Of course, the tremendous success of the description of the electroweak interactions in perturbation theory makes the Standard Model a very well tested theory in particle physics. However, this leaves us with the unsatisfactory situation of the Standard Model being good "for all practical purposes". At this level it is certainly not a well founded theory as e.g. QCD.

The conventional point of view today is to regard the Standard Model as only an effective theory to describe low energy physics. In this low energy limit perturbation theory works very well. However, due to triviality our theoretical description of the electroweak interactions is inherently incomplete. At some energy scale which depends on the Higgs boson mass the theory has to break down giving room for some -yet unknown- new physics. This new physics in some sense regulates the minimal Standard Model. Therefore, finding a non-perturbative regulator for a chiral gauge theory might give some hint about the theory "beyond the Standard Model". A non-perturbative chiral regulator would also be important to understand, whether a construction of an asymptotically free chiral gauge theory is possible [3, [4]. Not surprisingly 
then, the attempts of a non-perturbative definition of a chiral gauge theory have been numerous and in particular the search for lattice chiral fermions has been an intensive area of research in the last few years.

The outcome of all attempts to put the Standard Model on the lattice is, however, so far either negative or no convincing evidence of their success could be given. The stumbling block in constructing chiral gauge theories on the lattice are the famous nogo theorems by Karsten and Smit [1] and by Nielsen and Ninomiya [2], see also [5]. The assumptions of the nogo theorems are surprisingly mild. They require hermiticity, locality, and translational invariance of the underlying interaction. With these assumptions there will always be a clash between chiral gauge invariance and the possibility of a single Weyl fermion on the lattice. To each fermion with given chirality there will always be a doubler mode with the opposite handedness. This proliferation of fermions is deeply connected to the anomaly structure on the lattice. Either we will have chiral gauge invariance but then the doubler fermions appear and cancel the anomaly, or we lift the doublers by some mechanism and destroy chiral gauge invariance. It is worth to note that these theorems are claimed to hold also without refering to the lattice at all [2]. This is reminiscent of Adler's demonstration that it is impossible to find a gauge invariant regularization such that the axial current remains conserved [6]. It emphasizes the fact, that the question of regularizing chiral fermions non-perturbatively is not a problem for the lattice alone but one of principle.

To circumvent the nogo theorems one has then to violate some of the above assumptions at one stage. Although it is acceptable that at some intermediate step one of the above properties is missing, one clearly would like to recover them in the continuum theory as they are important ingredients for a sensible theory. There are numerous ways trying to find such violations and still recover the electroweak interaction in the continuum. For an overview I refer to several review talks in the annual Lattice proceedings [4, 0, 8, 9, 10]. In particular I would like to draw the reader's attention to the proceedings of the Rome workshop [1]] which contains virtually all approaches at that time. In [12] many of these attempts, the way how they tried to circumvent the nogo theorems and reasons why they did not work, are summarized.

One attempt of finding a method to simulate chiral fermions on the lattice was not covered in the Rome workshop. This was Kaplan's idea [13] of generating a chiral mode on a domain wall that is induced by a mass defect along an extra dimension. Imagine a scenario where we actually live in a 5-dimensional world. Assume that along the fifth dimension there is a defect generated by a fermion mass term that only depends on the fifth direction and has the form of a soliton. This will generate a domain wall and on this 4-dimensional wall we will find a chiral zeromode traveling along it [14, 15].

In 15] Callen and Harvey studied general anomaly descent relations [16] connecting fermion zeromodes on a domain wall and anomalies in even with parity anomalies in odd dimensions. In particular they demonstrated that the odd dimensional theory is anomaly free. Performing a Goldstone-Wilczek [17] type of calculation they showed that the anomaly in the gauge current 
due to the chiral zeromode on the domain wall is exactly cancelled by the divergence of the Chern-Simons current generated by the heavy fermions in the fifth dimension off the wall. That the Chern-Simons current is not divergence free is due to the fact that the mass defect changes sign when crossing the domain wall.

One interesting aspect of the Callan-Harvey analysis is, that it can be taken over to the lattice. Indeed, it has been pointed out already in [18 that this might lead to a realization of chiral lattice fermions .

Independently of [18] Kaplan suggested to use the domain wall setup to construct a chiral gauge theory on the lattice [13]. The hope is to produce a chiral theory on the 4-dimensional domain wall. Using the -in lattice QCD successful- Wilson mechanism, it can be expected that the dangerous doubler modes are decoupled. The difference to other approaches is that the 5 -dimensional theory one would start with is vectorlike from the very beginning thus avoiding problems with the nogo theorems. Through the Callan-Harvey mechanism the gauge anomaly would be reproduced on the domain wall while at the same time the 5-dimensional system remains anomaly free due to the mechanism of anomaly cancellation. Another attractive property of the domain wall model is, that also the fermion number current is anomalous on the 4-dimensional wall [13, 19], although, it is again conserved in the 5-dimensional system.

This promising picture evokes immediately some questions. Staying in a situation where the fermions are free or are at most only coupled to external gauge fields, we have a single Weyl fermion on the wall. Then how did we circumvent the nogo theorems? A first answer is that we violated translational invariance. But this is only true in the 5 -dimensional system. With respect to the 4-dimensional domain wall the system is translational invariant and still we have the single chiral fermion and the anomalies work out correctly.

The answer to this seemingly paradox can be given, if one looks at the finite lattice situation. There we have to introduce some kind of boundary condition in the extra direction. This leads - unavoidingly - to a second domain wall on which an additional zeromode appears. This mode has the opposite chirality as the one on the original wall. Both modes have an exponentially small overlap [13, 20, 21]. Therefore we are in full accordance with the nogo theorems. We indeed have the mode spectrum as demanded by the theorems. Due to the extra dimension we have just separated them such that they do not communicate. The whole setup is gauge invariant, but we created a loophole for the charges such that they can escape in the extra dimension leaving a chiral theory on the domain wall. For the infinite lattice the second domain wall is sent to infinity. That this picture is indeed correct, as long as we do not have dynamical gauge fields, could be demonstrated in [20, 22].

\footnotetext{
${ }^{1}$ There a domain wall system was studied in the context of a stacking fault in a PbTe-type crystal. Using the argumentation of Callan and Harvey the authors of ref. 18] discussed the apparent appearance of the parity anomaly. Using techniques similar to Callan and Harvey they showed that the complete 3 -dimensional condensed matter system is anomaly free. They proposed that the domain wall model may be used for solving the chiral fermion problem in lattice gauge theory. In particular they suggest to introduce the Wilson mechanism for lifting the doublers. However, they then gave up the domain wall model idea.
} 
There remains, of course, the crucial point what will happen when dynamical gauge fields are added. There have been two main lines of thought. The first one is to add 5-dimensional gauge fields giving the gauge fields in the fifth direction a coupling strength different from the 4-dimensional fields [13]. A number of papers investigated this scenario [23, 24, 25, 19, 26, 27]. The conclusion was that this setup would not lead to the desired chiral gauge theory. The main reason is that one would end up in the so-called layered phase where the system reduces to a standard 4-dimensional lattice system of Wilson fermions and no zeromodes appear. Hence the model becomes vectorlike and can not be used for a construction of a chiral lattice theory.

The alternative approach [28, 25, 29] is to leave the gauge fields strictly 4-dimensional and to confine it to only one of the walls. In this way only one of the domain walls would be gauged and we are left with a region in the extra direction which is gauged and its ungauged complement. For reasons that will become clear later we call the gauged region the "waveguide" region. At the boundary of the waveguide to the ungauged part in the fifth direction gauge invariance is broken. This leads to a situation where one studies either a gauge non-invariant model or a model which is gauge invariant but contains scalar fields at the boundaries of the waveguide. The scalar (or Stückelberg) fields serve to restore gauge invariance in much the same manner as the Standard Model is made gauge invariant. Adding a scalar field that couples to the fermions suggests, to also introduce a Yukawa-coupling $y$. It can therefore be expected that fermion masses are generated via the Higgs mechanism through the Yukawa-coupling of the fermions to the scalar vacuum expectation value. These fermion modes will live at the waveguide boundary. The crucial question is, whether these states can be decoupled such that we are left with only the gauged and ungauged domain wall zeromodes separated from each other in the fifth dimension.

One may argue that such a scenario is condemned to fail from the very beginning. The reason being that approaching the phase transition from a spontaneously broken to a symmetric phase the vacuum expectation value $v$ approaches zero giving rise to small fermion masses which may be interpreted as mirror fermions. In particular these masses should be zero in the symmetric phase. Thus they would not decouple. However, investigations of Yukawa models on the lattice revealed a surprisingly rich phase structure at large values of the Yukawa-coupling [30]. In particular a so-called strong coupling region could be identified [31, 32, 33]. In this region the Yukawa-coupling becomes so strong that the fermions combine with the scalar fields to form massive bound states with masses at the cut-off. If such a region would exist also in the domain wall model, the fermions at the waveguide boundary could be made heavy and would decouple. We then would be left with a single chiral zeromode bound to the domain wall.

Another argumentation which would make the domain wall model fail is that the charge due to this chiral zeromode will flow off the wall transported by the Chern-Simons current and would have to be deposited somewhere. The most easy scenario to imagine is that there are additional fermion zeromodes that induce a current absorbing this charge [34]. However, due to our waveguide setup, the gauge fields have to stop somewhere in the fifth dimension. At this 
point we will have a change of the gauge field that can induce a Wess-Zumino current. This current can give rise of cancelling the anomaly.

From these considerations it is clear, that the waveguide model can not be ruled out by simple arguments. The question whether the domain wall approach would be successful or not becomes a dynamical one and a numerical simulation becomes necessary. Such a simulation for a 3-dimensional setup was performed in [25]. There the weak Yukawa-coupling region, where the fermion masses are proportional to $v$ and therefore vanish in the symmetric phase, could be established. For large values of the Yukawa-coupling the results of this simulation were compared with Yukawa models that have been investigated earlier and that do or do not show a strong coupling behaviour. Strong evidence was collected that in the domain wall model a phase with the strong coupling behaviour, as described above, does not exist. This was confirmed by the results of a strong coupling expansion which revealed a new phase with weak coupling behaviour at large $y$. It has to be concluded therefore that in the domain wall model physics is governed by a weak coupling behaviour at all values of $y$ thus not leading to a chiral theory.

In this review we will in the following discuss the domain wall model in more detail. Throughout this paper the fermions are thought to be taken in an anomaly free representation. After introducing the fermion doubling problem and how it may be solved by the domain wall model we will continue to give an explicit demonstration of the Callan-Harvey picture of charge flow on the lattice. We then present the model with the scalar fields added. We discuss a large $y$ expansion in this model and the results of the numerical simulations. We will present the suggestion that the extra dimension can be held strictly infinite. We end with a possible prospect of the domain wall model for simulating QCD and give a summary and conclusions.

\section{Free fermions, doubling and a possible escape}

In this section the problem of fermion doubling on the lattice [35] is illustrated and the idea of domain wall fermions introduced. The discussion will be for two dimensions only and the Hamiltonian formalism will be used. The results obtained in this simple setting show already the essential features and are readily generalized to arbitrary dimensions. In this section we will indicate the lattice spacing $a$ explicitly in the formulae. Otherwise $a$ is set to one throughout the paper. We start our discussion with the 1-dimensional Hamilton operator for free fermions which -in the continuum- may be written as $H_{c}=-\sigma_{1}\left(\not \partial+m_{0}\right)$. The operator $\not \partial=\sigma_{2} \partial / \partial x$ acts only on the $x$ coordinate and the $\sigma$ 's are the usual Pauli matrices. $H_{c}$ describes a single fermion of mass $m_{0}$ obeying the relativistic dispersion relation $E^{2}=p^{2}+m_{0}^{2}$. A "naive" discretization of $H_{c}$ on the infinite lattice would be

$$
H=-\sigma_{1}\left[\sigma_{2} \partial_{x}+m_{0}\right]
$$


where $\partial_{x}$ now denotes the lattice derivative

$$
\partial_{x}=\frac{1}{2 a}\left[\delta_{x, x+\mu}-\delta_{x, x-\mu}\right]
$$

$a$ denotes the lattice spacing and $\mu$ is a displacement by $a$ in a given direction on the lattice. In our 1-dimensional example $\mu=a$.

By Fourier transformation, the particle energies are easily obtained as

$$
E= \pm \sqrt{\frac{1}{a^{2}} \sin ^{2}(a k)+m_{0}^{2}}
$$

with $\mathrm{k}$ lying in the Brillouin zone $-\pi<a k \leq \pi$. For $k a \ll \pi$, the naive continuum limit, $a \rightarrow 0$, reproduces the above relativistic dispersion relation $E^{2}=k^{2}+m_{0}^{2}$. However, for momenta $a k \approx \pi$, we find again $E^{2}=k^{2}+m_{0}^{2}$ and obtain another fermion with mass $m_{0}$. Moreover, while the fermion at the origin of the Brillouin zone at $a k \approx 0$ is a right moving particle as can be seen from the group velocity $d E / d k$, the one appearing at the corner of the Brillouin zone at $k \approx \pi / a$ is a leftmover since the sin function changes its sign there. For massless fermions this amounts to find two chiral fermions with opposite chirality quite in contrast to the single Weyl fermion we would have started with in the continuum. This is the famous doubling phenomenon for lattice fermions. The fact that one finds opposite chirality fermions at the different corners of the Brillouin zone is a consequence of the "doubler symmetry" on the lattice [36]. This symmetry transformation is represented by a matrix

$$
M=M_{1} M_{2} ; M_{j}=i \sigma_{3} \sigma_{j}
$$

If $\psi_{k}$ is an eigenfunction of the Hamiltonian in momentum space, this transformation exchanges the corners of the Brillouin zone, $\psi_{k}=M \psi_{k+\pi}=-\sigma_{3} \psi_{k+\pi}$. Thus, the mode at the opposite corner of the Brillouin zone has a flipped chirality. The doubler symmetry gives in general dimensions an equal number of left and right handed particles. As the representation of the corresponding doubler symmetry group is irreducible, doublers have to appear in the fermionic lattice spectrum as long as the doubler symmetry is unbroken.

The solution of the doubling problem as proposed by Wilson [5] is to break the doubler symmetry by adding a second derivative term to the Hamiltonian

$$
H=-\sigma_{1}\left[\sigma_{2} \partial_{x}+m_{0}-r \Delta_{x}\right]
$$

with

$$
\Delta_{x}=\frac{1}{2 a}\left[\delta_{x, x+\mu}+\delta_{x, x-\mu}-2 \delta_{x, x}\right]
$$

The spectrum becomes

$$
E^{2}=\frac{1}{a^{2}} \sin ^{2}(a k)+\left(m_{0}+\frac{r}{a}(1-\cos (a k))\right)^{2} .
$$


The mass of the fermions are now given in the $a \rightarrow 0$ limit as

$$
m=m_{0}+2 \frac{r}{a} n_{\pi}
$$

with $n_{\pi}=0$ for the origin of the Brillouin zone at $a k \approx 0$ and $n_{\pi}=1$ for the corner of the Brillouin zone at $a k \approx \pi$. Therefore, we obtain for the fermion at the origin of the Brillouin zone again the continuum dispersion relation but the fermions at the corners get an additional piece proportional to $1 / a$. They will become infinitely heavy as $a \rightarrow 0$ and decouple from the spectrum. In general dimensions $n_{\pi}$ counts the number of $\pi^{\prime} s$ at the different corners of the Brillouin zone.

Unfortunately, there is a price to pay for getting rid of the extra unwanted fermions. Imagine, we add dynamical gauge fields to the Hamiltonian (5) by making the lattice derivatives (2), (6) gauge covariant. The Wilson term clearly acts as a mass term so that even if we take the fermion mass $m_{0}$ to zero, we still loose chiral gauge invariance. Although chiral symmetry can be restored in QCD by carefully tuning the bare parameters to some critical value, this appears to be a disaster for the electroweak interactions.

Indeed, for vectorlike gauge theories as QCD, weak coupling perturbation theory revealed the restoration of chiral symmetry for the Green functions in the continuum limit [1], 37]. This opened the road for lattice simulations of QCD because - though technically very challenging- no problem of principle remains. For a chiral gauge theory using Wilson's approach [35, 7, 38], weak coupling perturbation theory [39] showed no success in obtaining the desired target theory and the doublers could not be decoupled. This pessimistic picture was strengthened by results from lattice simulations which showed that for weak coupling the doublers remain in the spectrum [11, 40]. Also the hope of decoupling as a non-perturbative effect failed [41, 42]. It seems that in order to obtain a chiral gauge theory one has to be more ingenious than in QCD.

\subsection{Domain wall fermions}

A possible escape from the doubling problem is due to D. Kaplan who suggests to send the dangerous modes into an extra dimension. It is known since a long time that there exists a chiral zeromode solution of the continuum Dirac equation in presence of a soliton [14. The idea to use such a kind of solution to construct chiral lattice fermions is to regard our 4-dimensional world as a domain wall embedded in 5 dimensions. The interface is induced through a mass defect in form of a soliton. To stay in our 2-dimensional setup of the previous section we will study a 3-dimensional system. Along the third extra dimension denoted by $s$, a mass defect is introduced through

$$
m(s)=\left\{\begin{array}{ll}
-m_{0} ; & s \rightarrow-\infty \\
+m_{0} ; & s \rightarrow+\infty
\end{array} .\right.
$$

The concrete form -aside that it has to be monotonic- of the mass is not very important. It is easy to show that in this situation there exist solutions $\Psi_{ \pm}$which are energy eigenstates of the 
continuum Hamiltonian

$$
\begin{gathered}
H_{c}=-\sigma_{1}\left[\sigma_{2} \partial(x)+\sigma_{3} \partial(s)+m(s)\right], \\
\Psi_{ \pm}=e^{i p_{x} x} \Phi_{ \pm}(s) u_{ \pm}
\end{gathered}
$$

with

$$
\Phi_{ \pm}(s)=\exp \left( \pm \int_{0}^{s} m\left(s^{\prime}\right) d s^{\prime}\right)
$$

and $u_{ \pm}$a chiral eigenstate, $\sigma_{3} u_{ \pm}= \pm u_{ \pm}$. Only the function $\Phi_{-}(s)$ corresponds to a normalizable solution. This solution describes a chiral zeromode traveling along the interface. It is bound to the wall and falls off exponentially with growing distance from the wall.

Translating this model to the -infinite- lattice we choose a step function for the mass

$$
m(s)=m_{0} \theta(s) ; \quad \theta(s)=\left\{\begin{array}{ll}
-1 ; & s \leq-a \\
0 ; & s=0 \\
+1 ; & s \geq a
\end{array} .\right.
$$

The continuum derivatives are again replaced by finite lattice differences and the Hamiltonian reads now

$$
H=-\sigma_{1}\left[\sigma_{2} \partial_{x}+\sigma_{3} \partial_{s}+m(s)\right] .
$$

Imposing an ansatz similar to the continuum solution

$$
\Psi_{ \pm}=e^{i k x} \Phi_{ \pm}(s) u_{ \pm}
$$

we find again a normalizable solution

$$
\Phi_{-}(s)=e^{-\mu_{0}|s|},
$$

with $\sinh \mu_{0}=m_{0}$. This is a solution that is bound to the domain wall, it is chiral and describes plain waves along the direction of the domain wall. Unfortunately, there exists another solution which only appears on the lattice

$$
\Psi_{+}=e^{i k x} \Phi_{+} u_{+}
$$

with

$$
\Phi_{+}(s)=(-1)^{s} \Phi_{-}(s) .
$$

This solution describes again a massless fermion traveling along the domain wall, it is also bound to it and has opposite chirality as compared to the solution $\Phi_{-}(s)$, eq.(16). In other words it is a doubler fermion in the s-direction! Even worse, also the doubler mode in the $x$-direction from the corner of the Brillouin zone is a solution. Therefore the spectrum on the domain wall consists of two chiral fermions with positive chirality and their two doublers -one in the $s$ and one in the $x$ direction- with opposite chirality.

What we have achieved so far is instead of removing the doubler modes we have created one in addition. But what if we now try to apply Wilson's trick adding a higher derivative term? 
The 3-dimensional model we started with is vectorlike as in QCD and we saw that there the Wilson term is harmless. The Hamiltonian becomes

$$
H=-\sigma_{1}\left[\sigma_{2} \partial_{x}+\sigma_{3} \partial_{s}+m(s)-r\left(\Delta_{x}+\Delta_{s}\right)\right]
$$

Let us set the Wilson coupling $r=1$ for the moment and again try an exponential ansatz for the transverse wavefunction $\Phi$ keeping plane wave solutions in the $x$-direction

$$
\Phi_{ \pm}(s \pm a)=-k_{e f f}(s) \Phi_{ \pm}(s)
$$

with an effective momentum

$$
k_{e f f}(s)=m_{0} \theta(s)-1-F(k) ; \quad F(k)=1-\cos (a k) .
$$

In order to obtain normalizable solutions, the functions $\Phi$ have to decrease with growing $|s|$ on both sides of the wall. This implies the following conditions to be fulfilled

$$
\begin{aligned}
& \Phi_{+}:\left|k_{e f f}\right|>1 \text { for } s<0, \quad\left|k_{e f f}\right|<1 \quad s>0 \\
& \Phi_{-}:\left|k_{e f f}\right|<1 \text { for } s<0, \quad\left|k_{e f f}\right|>1 \quad s>0 .
\end{aligned}
$$

One recognizes that only one of the solutions is normalizable namely $\Phi_{-}$and that we have to throw away the $\Phi_{+}$solution. Thus we are left with only one zeromode solution and we got rid of the doubler mode in the $s$-direction (18). Since the doubler mode in the $x$-direction is decoupled by the usual Wilson mechanism we remain with a single chiral fermion solution as in the continuum. This chiral zeromode is bound to the domain wall and exponentially decreases going away from the wall. The normalizability condition (22) requires

$$
0<m_{0}-F(k)<2 .
$$

This means that for a given value of the domain wall height $m_{0}$ the chiral zeromode does not exist for all momenta but only up to some critical momentum $k_{c}$. At this value the fermion ceases to be chiral and vanishes in a band of heavy modes. This behaviour is the important ingredient of the domain wall model. The chiral zeromode only exists as a low energy phenomenon. For large values of the momentum it becomes heavy and is not distinguishable from the doubler modes. It therefore does not appear again as a low momentum state at some other corner of the Brillouin zone.

In [21] the zeromode solutions for general $m_{0}$ and $r$ values were computed. There the 3-dimensional Dirac-operator was studied. Therefore the momentum space becomes 2-dimensional. The normalizability condition led to a very peculiar zeromode spectrum (see also fig.1 in [21]). For $m / r \leq 0$ no chiral fermions exist. Increasing $m / r$ a chiral fermion is found with the critical momentum given by $m_{0}=r F(k)$. Increasing $m / r$ further the value of the critical momentum keeps growing, too. This continues until at $m / r=2$ the maximal critical momentum is reached. For $m / r>2$ the chiral zeromode at the origin of the Brillouin zone $\vec{k}=(0,0)$ is lost. Instead 
new zeromodes appear at the corners of the Brillouin zone $\vec{k}=(\pi, 0)$ and $\vec{k}=(0, \pi)$. They have the opposite chirality as the one at $k \approx 0$. The critical momenta of these new zeromodes are in the interval determined by $m_{0}=r F(k)$ for the lower and $m_{0}=r(F(k)+2)$ for the upper momentum interval boundary. Increasing $m / r$ even further we loose these modes again for $m / r>4$ but get a new zeromode at $\vec{k}=(\pi, \pi)$ with flipped chirality. Finally, for $m / r>6$ the chiral zeromodes disappear completely. Depending on the bare parameters of the domain wall model one can therefore generate a chiral zeromode spectrum as needed.

\subsection{The domain wall model on the finite lattice}

The interesting question is, of course, whether the above scenario can be realized on a finite lattice. There one has the additional complication that some kind of boundary condition has to be imposed which necessarily induces a second domain wall. In the following we let the lattice have finite extensions $L_{s}$ in the $s$ and $L$ in the $x$-direction and have $s \in\left[1, L_{s}\right], x \in[1, L]$. The domain wall mass will be chosen

$$
m(s) \equiv \sinh \left(\mu_{0}\right) \theta(s) ; \theta(s)=\left\{\begin{array}{ll}
-1 & 2 \leq s \leq \frac{L_{s}}{2} \\
+1 & \frac{L_{s}}{2}+2 \leq s \leq L_{s} \\
0 & s=1, \frac{L_{s}}{2}+1
\end{array} .\right.
$$

The finite lattice Hamiltonian including the Wilson term reads

$$
H=-\sigma_{1}\left[\sigma_{2} \partial_{x}+\sigma_{3} \partial_{s}+m(s)-r\left(\Delta_{x}+\Delta_{s}\right)\right]
$$

Due to the finite lattice extension, we have to specify some sort of boundary condition which will be chosen to be periodic in the $s$ and antiperiodic in the $x$-direction. The Hamiltonian (25) can be reduced to only depend on $s$ by imposing plane wave solutions in the $x$-direction. One obtains, setting $a=1$,

$$
\left.H=-\sigma_{1}\left[\sigma_{2} \sin (k)+\sigma_{3} \partial_{s}+m(s)+r(\cos (k)-1)-r \Delta_{s}\right)\right],
$$

where the momenta $k$ are now discretized,

$$
k=\frac{\pi}{L}\left(n+\frac{1}{2}\right), n=0, \ldots, L-1 .
$$

This choice of the momenta $k$ corresponds to antiperiodic boundary conditions which will also be chosen in numerical investigations presented later. They become necessary in numerical simulations to avoid exact zeromodes which render the simulation algorithms impractical.

In this form the energy eigenvalues and the corresponding eigenfunctions for a given value of $k$ can be obtained by diagonalizing the Hamiltonian which is a $2 L_{s} \otimes 2 L_{s}$ matrix numerically. In this way one gets the momentum dependent eigenvalues $\lambda_{ \pm k}$ and their corresponding wavefunctions. The eigenvalues come in \pm pairs. In fig.1a the wavefunctions belonging to the lowest positive eigenvalue $\lambda_{0}$ and its negative partner are plotted. One clearly sees that the second 


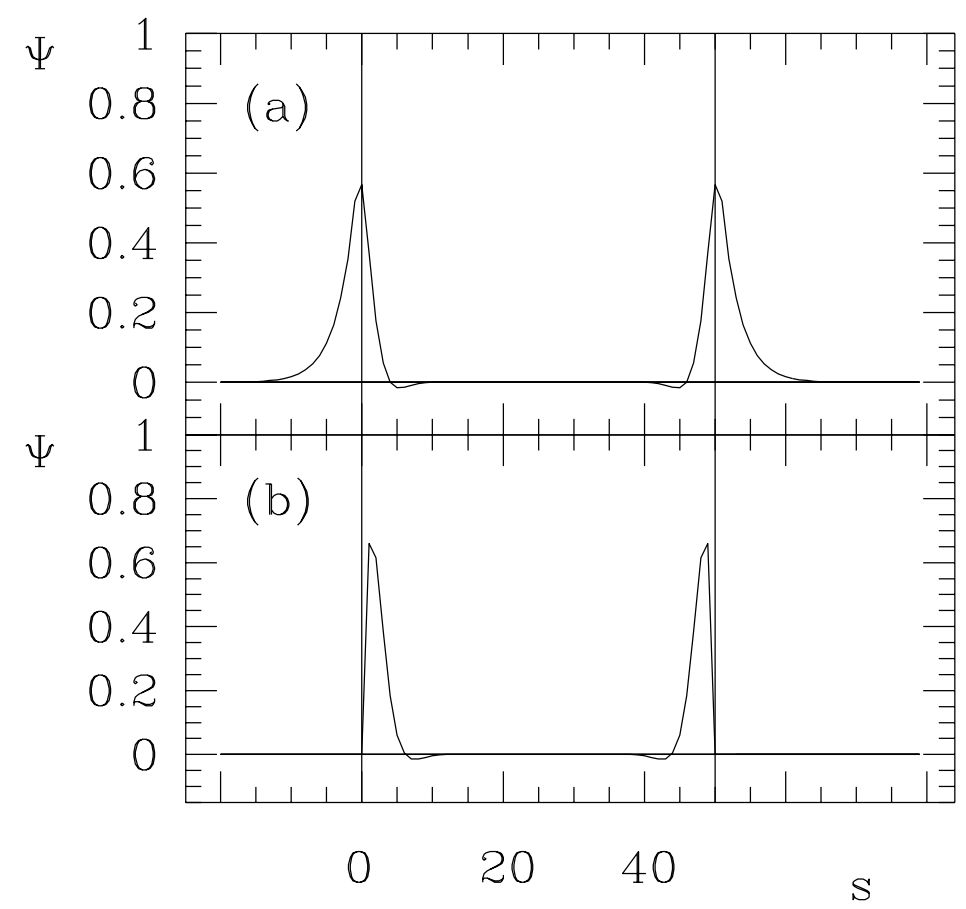

Figure 1: The zeromode wavefunctions as obtained from the finite lattice Hamiltonian (26). The domain wall mass $\mu_{0}=1$, the Wilson coupling $r=1$ and the lattice size in the $x$-direction is $L=32$. The horizontal lines indicate the position of the domain walls. Fig. 1a is the domain wall model with an extension of $L_{s}=100$ in the extra dimension. Fig. $1 \mathrm{~b}$ is the variant of this model with open boundary conditions and an extension of $L_{s}=50$ only. Both figures show that the zeromode wavefunctions are sharply localized on the walls and fall off very rapidly with growing distance from the wall.

domain wall generates an additional solution which is absent on the infinite lattice. The nice thing is that both solutions are located on each of their domain walls at $s=1$ and $s=L_{s} / 2+1$. They have opposite chirality and fall off exponentially going away from the domain wall having only an exponentially small overlap $\propto e^{-\mu_{0} L_{s}}$.

In fig.2a the lowest and the next to lowest positive eigenvalues are plotted as a function of the lattice momenta $\mathrm{k}$ corresponding to antiperiodic boundary conditions. One observes the existence of the critical momentum $k_{c}$. The lowest eigenvalue $\lambda_{0}$ follows the dispersion relation of a free lattice fermion $\sin (k)$ up to the critical momentum where it vanishes in a band of larger eigenvalues. In fig. 2a also the next lowest positive eigenvalue $\lambda_{1}$ is plotted. For low momenta where $\lambda_{0}(k)=\sin (k)$ it stays at the order of the lattice cut-off exhibiting a large gap to the lowest eigenvalue. It stays almost constant as a function of the momenta until at $k_{c} \approx 1$ it combines with $\lambda_{0}$. Fig. $2 \mathrm{~b}$ shows that the mode corresponding to the lowest positive eigenvalue 
really is a chiral fermion. The ratio build from the wavefunction $\psi_{0}$ belonging to $\lambda_{0}$

$$
R=\frac{<\bar{\psi}_{0} \psi_{0}>}{<\bar{\psi}_{0} \sigma_{1} \psi_{0}>}
$$

indicates the chirality of a mode. It is zero when $\psi$ is a chiral eigenstate and becomes non-zero if chirality is lost. The figure shows that this happens exactly at the place where the gap $\lambda_{1}-\lambda_{0}$ in fig. 2 a shrinks to zero which is at $k_{c}$.

\section{The anomaly}

The fermion spectrum we have obtained in the previous section looks very promising. We have two Weyl fermions on the finite lattice living on each of the domain walls. They are separated in the extra dimension and have an only exponentially small overlap. Besides the chiral fermions we have the heavy doubler modes on the walls and additional heavy fermions off the wall with masses at the order of the cut-off. For free domain wall fermions the low energy physics on both walls appears therefore to describe a chiral theory.

Let us assume that adding gauge fields will not destroy this picture. This is, of course, a strong assumption but, as we will see, it is at least justified for weak external gauge fields. Introducing dynamical gauge fields is much more problematic and will be discussed later. A (2-dimensional) physicist living on one of the walls knowing nothing about he extra dimension would eventually discover "his" Standard Model - the 2-dimensional chiral Schwinger model. He would also discover the chiral anomaly and wonder how he could regularize his chiral theory. If the domain wall model is able to succeed in producing a chiral gauge theory on a wall as the low energy limit of the 3-dimensional model, it should as a first step generate the correct anomaly structure for external gauge fields. Therefore the computation of the anomaly in the 2-dimensional Schwinger model is a first crucial step in testing the domain wall approach. Since at the present stage the gauge fields are only external, we might select our physical wall by hand. It is therefore irrelevant that the gauge fields connect both chiral zeromodes. What we are aiming at, is to get a picture of the charge flow in the finite lattice model containing both walls. For the following calculations we use a variant of the domain wall model as introduced by Shamir [43] and follow [44] to compute the axial charge in the Hamiltonian formalism from the spectrum flow.

Shamir's variant is to not let the mass change its sign across the second domain wall but to cut it off completely and introduce open boundary conditions instead. The system can be regarded as being in a box with infinitely high walls at the ends of the extra dimension. The Hamiltonian analysis of this situation is very similar to the above discussion 43. In this setup the chiral zeromodes appear as surface modes on the walls with opposite chirality on the two borders. We show in fig.1b the zeromode spectrum of the Hamilton operator with open boundary conditions in the $s$-direction. As before we keep antiperiodic boundary conditions 


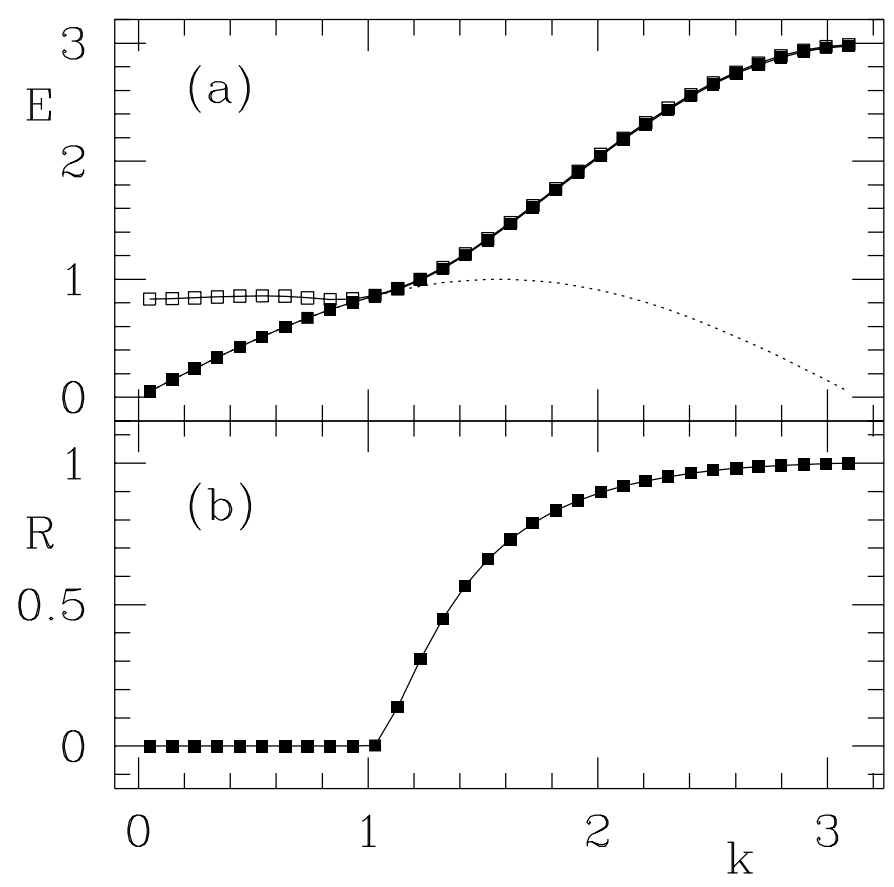

Figure 2: The lowest and next to lowest positive eigenvalues are plotted in fig.2a against the lattice momenta $k=\pi(n+0.5) / L$ with $n=0, \ldots, 31$ corresponding to a lattice of 32 points in the x-direction with anti periodic boundary conditions. The parameters are the same as in fig.1. The dotted line is the energy of a free massless fermion on the lattice, $\sin (k)$. One nicely observes that the lowest eigenvalue $\lambda_{0}$ (full squares) follows the $\sin (k)$ behaviour whereas the next mode $\lambda_{1}$ (open squares) is at the order of the cutoff and stays almost constant as a function of $k$. At a critical value of the momentum $k_{c} \approx 1$ the gap $\lambda_{1}-\lambda_{0}$ shrinks to zero. At this point the lowest mode departures from the sin behaviour. Fig. $2 \mathrm{~b}$ shows that the mode corresponding to $\lambda_{0}$ is really chiral. Plotted is the ratio $R=\left\langle\bar{\psi} \psi>/<\bar{\psi} \sigma_{1} \psi>\right.$ for the eigenfunction of $\lambda_{0}$. $R$ is zero if $\psi$ is a chiral eigenfunction and deviates from zero if the chirality is lost. $R$ becomes non-zero at exactly the value of $k$ where the two modes in fig.2a combine, i.e. at $k_{c}$. 
in the $x$-direction and choose the same parameters as in fig.1a. The figure shows the surface modes which are again separated in the extra dimension. They have only exponentially small overlaps and are both eigenstates of $\sigma_{3}$, the chiral operator. It is remarkable how similar they are in shape compared to the domain wall zeromodes depicted in fig.1a. The boundary version of the domain wall model has the advantage that the lattice in the $s$-direction is only half that of the domain wall model. This might lead to an improvement in practice if one thinks of numerical simulations. This would be the case in particular when the domain wall fermions are going to be used for simulations of QCD, a prospect we will discuss later.

To see that there really flows an anomalous current along the domain wall we apply a time dependent external field in the $x$-direction. We introduce the gauge covariant lattice derivatives

$$
\partial_{x}(U)=\frac{1}{2}\left[U_{x, \mu} \delta_{x, x+\mu}-U_{x-\mu, \mu}^{*} \delta_{x-\mu, x}\right]
$$

and

$$
\Delta_{x}(U)=\frac{1}{2}\left[U_{x, \mu} \delta_{x, x+\mu}+U_{x-\mu, \mu}^{*} \delta_{x-\mu, x}-2 \delta x, x\right] .
$$

Here $U$ denotes the usual compact representation of the gauge field. In this review we will for all discussions only use $U(1)$ gauge fields. Then $U$ is a phase, $U_{x, \mu}=e^{\left\{i \alpha_{x, \mu}\right\}}$ and is related to the continuum gauge potential $A_{\mu}(x)$ by $e^{i q A_{\mu}(x)}$. The gauge fields will be chosen to be only 2 -dimensional and identical on every $s$-slice. Although this would lead to an interaction of the surface modes when the gauge fields were fully dynamical, this is not a problem for only external gauge fields because in this case a domain wall can be singled out by hand.

The Hamilton operator becomes

$$
H=-\sigma_{1}\left[\sigma_{2} \partial_{x}(U)+\sigma_{3} \partial_{s}+m(s)-r\left(\Delta_{x}(U)+\Delta_{s}\right)\right]
$$

If we think of $H$ being written in terms of creation and annihilation operators $\hat{\psi}^{\dagger}$ and $\hat{\psi}$, it is gauge invariant under a gauge transformation

$$
U_{x, \mu} \rightarrow g_{x} U_{x, \mu} g_{x+\mu}^{*} ; \psi_{x} \rightarrow g_{x} \psi_{x}, g \in U(1)
$$

We will choose an external field that is constant in space and varies only in time. For adiabatic external fields we expect then from the continuum

$$
\begin{aligned}
Q_{3}(t) & \equiv \frac{q}{2 \pi} \int_{0}^{t} d t^{\prime} \int d x \epsilon_{\mu \nu} F^{\mu \nu}\left(x, t^{\prime}\right) \\
& =-\frac{q}{2 \pi} L \int_{0}^{t} 2 d t^{\prime} \frac{\partial}{\partial t^{\prime}} A\left(x, t^{\prime}\right) \\
& =-2 q \frac{L}{2 \pi} A_{1}(t) .
\end{aligned}
$$

Here we identify the gauge potential $A_{1}(t)=q \alpha(t)$, with $q=1$ the gauge charge and $Q_{3}$ denotes the (axial) charge. Due to the gauge invariance (32) the Hamiltonian (31) depends only on the product of all the gauge links. Thus the gauge potential $U=e^{i \alpha}$ becomes a constant 
global phase factor acquired by a fermion traveling around the finite lattice system. The lattice Hamiltonian reads in momentum space

$$
\left.H=-\sigma_{1}\left[\sigma_{2} \sin (k-\alpha)+\sigma_{3} \partial_{s}+m(s)+r(\cos (k-\alpha)-1)-r \Delta_{s}\right)\right]
$$

This is the free Hamiltonian with momenta shifted by the phase $\alpha$.

A definition of the axial charge is quite arbitrary on the lattice 45. Basically one has to fulfill that opposite charge has to be assigned to the two chiral modes. Defining the vacuum as the state with all negative energy levels filled, we can define a measure of the charge by

$$
Q_{3}=\frac{1}{L_{s}-1} \sum_{q, s}\left(L_{s}-1-2 s\right) \psi_{s, q}^{\dagger} \psi_{q, s}
$$

where the sum goes over the one particle wavefunctions $\psi_{s, q}$ above the vacuum. The definition of $Q_{3}$ in (35) has the property to be one, if the mode is located exactly to the left domain wall and minus one if it is located exactly to the opposite wall. For heavy modes that are smeared over the lattice the contribution to $Q_{3}$ is zero. In [44] the charge $Q_{3}$ was measured. The result is shown in fig. 3 where we plot the charge $Q_{3}$ as a function of the flux $\alpha$. If we measure $\alpha$ in units of $2 \pi / L$ the anomaly equation (33) becomes $Q_{3}=-2 \alpha$. This behaviour can indeed be observed in fig. 3 .

If the external field would really be adiabatic, the turning on of the field would be so slow that the surface modes on the walls can tunnel through the extra dimension, exchanging there roles. This would happen at $\alpha=1 / 2$. At this point we will have a change of the zeromode spectrum (see section 2.1). A filled level on one of the boundaries is just about to become the positive energy particle and an empty level is about to drop in the sea. We would observe a jump in the charge by -2 and the system would relax to the ground state at $\alpha=1$ resulting in a zero net charge. This is indicated in fig. 3 as the solid line.

We saw, however, that the wavefunctions of the surface fermions are sharply peaked at the boundary, see fig.1b. Therefore their overlap is exponentially small resulting in a very tiny tunnel energy $\delta$. For the situation in fig. $3, \delta \approx 10^{-9}$. Thus $1 / \delta$ is the largest timescale in the

problem. Instead of tunneling of both surface states, a particle-hole pair is created and the charge keeps decreasing towards $Q_{3}=-2$ at $\alpha=1$. This is represented as the stars in fig.3.

\subsection{The resolution of a puzzle}

For the reader who knows of the extra dimension there appears to be a puzzle from the results of the previous section. In every step of the above discussion the odd dimensional model was gauge invariant and vectorlike. We are also in full accordance with the nogo theorems in that there are an equal number of left and right handed fermions. The only trick to circumvent them is that these modes are separated in the extra dimension. In fact, the whole idea was to study a vector theory in order to be able to use the Wilson mechanism in close analogy to lattice QCD to decouple the doublers. 


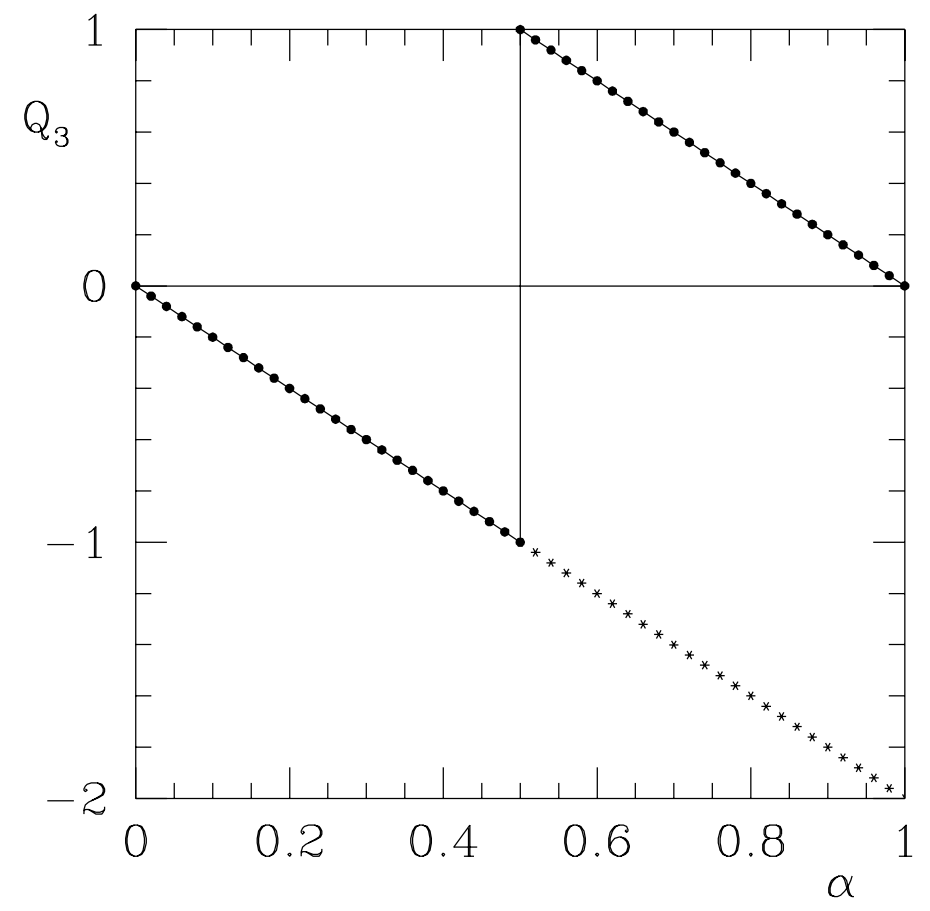

Figure 3: The charge $Q_{3}$ as function of the "flux" $\alpha$. The slope is $\approx-2$ as expected from the continuum anomaly equation.

So, how can there be then anomalous charge creation? The resolution of this puzzle are the anomaly descent relations [15, 16]. Callan and Harvey pointed out that in an odd dimensional theory with a mass defect in form of a domain wall the heavy modes residing in the extra dimension off the domain wall do not entirely decouple but produce a Chern-Simons term in the effective action when they are integrated out. This action generates a Chern-Simons current which is very similar to a Hall-current in solid state Physics. It flows along the extra dimension and perpendicular to the gauge current induced by the external field. The ChernSimons current is responsible for a charge transport in the extra dimension from one of the walls to the other. The strength of the Chern-Simons current is different on the two sides of the wall. Therefore the Chern-Simons current is not divergenceless. Indeed, as could be shown by Callan and Harvey, the divergence of this current is exactly equal in strength to the anomaly on the wall. This mechanism makes the even dimensional observer think that charge just "disappears" or is "generated" out of nowhere. The total 3-dimensional system is anomaly free and contains an equal number of left and right handed fermions just as demanded by the nogo theorems.

With these remarks we leave now the Hamiltonian formalism to discuss the domain wall model and go to the euclidean action formulation in which simulations have to be performed in the end. We start with an explicit lattice computation of the Chern-Simons current, confirming the continuum picture of the anomaly cancellation by Callan and Harvey being realized on the 
lattice, too.

\section{Euclidean lattice action for domain wall fermions}

The Hamilton formalism for free domain wall fermions discussed in the previous two sections led to some remarkable properties of the domain wall model in its wall-anti-wall realization on a finite lattice. There is a single Weyl fermion bound to the domain wall with its chiral partner living on the anti domain wall separated through the extra dimension. All other doubler modes are heavy and decoupled by means of the Wilson mechanism. From the spectral flow we saw that the charge on the domain wall very closely followed the continuum anomaly equation. These properties are certainly very essential building blocks should the model succeed in describing a chiral gauge theory on the lattice. Would it have failed at this point, there would have been no reason to continue with its investigation. The considerations of the last sections can be regarded as some necessary first checks every lattice chiral fermion proposal should pass in order to have a chance of being successful.

Our aim is a non-perturbative understanding of a chiral lattice model. This will most probably involve a numerical simulation at one stage. Such simulations are done by evaluating the - euclidean- path integral

$$
Z=\int \mathcal{D} \psi \mathcal{D} \bar{\psi} \mathcal{D} U e^{S_{G}+S_{F}}
$$

with $S_{G}$ the gauge field action and $S_{F}$ the fermionic part. We will not give the explicit form of both actions. Various alternatives will be discussed in the next section when possible additions of dynamical gauge fields to the domain wall model are discussed. Since we are aiming at a euclidean lattice description, we switch now the language going from the Hamiltonian formalism to the path integral formulation of the model. We start by performing an explicit calculation of the Chern-Simons current on the lattice. We will in the following stay in the $d=2+1$ dimensional setting and will always have abelian gauge fields.

In their continuum calculation, Callan and Harvey pointed out [15] that in a situation with a mass defect in odd dimensions there is a Chern-Simons current which is generated by the heavy fermions living far off the wall. This current has opposite sign on the two sides of the wall and its divergence is hence non-zero. In fact, Callan and Harvey computed the divergence of the Chern-Simons current and found that it exactly reproduces the coefficient of the anomaly generated by the chiral zeromode on the domain wall. It is this mechanism which gives hope that the domain wall model is able to provide the anomalies which are needed for a reproduction of the anomaly structure in the Standard Model.

The Callan-Harvey analysis can be considered to be incomplete in two aspects. ( $i$ ) The computation of Callan and Harvey has been done far off the wall and a computation also close to the wall would be desirable. (ii) The Chern-Simons coefficient is regulator dependent and it should be computed in different regularizations. In fact, the continuum calculation of Callan

and Harvey was done without imposing a regularization at all. We will improve this situation 
by presenting an analysis of the Chern-Simons current that is valid also arbitrary close to the wall [46] and give an explicit lattice computation of the Chern-Simons current [47, 22, 48].

(i) The effective Chern-Simons action is obtained when the heavy fermion modes are integrated out. The gauge variation of this action then leads to the Chern-Simons current the coefficient of which can be calculated in the low energy limit. This program has been gone through in the continuum by Callan and Harvey. However, their computation was done far off the domain wall where the mass defect can be considered as constant. It would be obviously desirable to have an analogous calculation also arbitrary close to the wall $\mathrm{f}$.

Such a continuum calculation has recently been given in 46]. Choosing the mass defect in the form

$$
m(s)=m_{0} \tanh \left(m_{0} s\right)
$$

we search for solutions of the eigenvalue equation in Minkowski space $\sigma_{1}\left\{i \sigma^{\mu} \partial / \partial x_{\mu}+m(s)\right\} \psi_{\lambda}=$ $\lambda \psi_{\lambda}, \mu=1,2,3$. The problem of solving this equation for the 3 -dimensional free domain wall system can be transformed to that of a quantum mechanical scattering problem with a modified Pöschl-Teller potential which can be solved exactly. Thus we not only get the chiral zeromode solution discussed in section 2 , but also all the heavy fermion solutions. From the wavefunctions $\psi_{\lambda}$ and the eigenvalues $\lambda$ the propagator $G\left(z, z^{\prime}\right), z=(x, t, s)$ can be calculated

$$
G\left(z, z^{\prime}\right)=\sum_{\lambda} \frac{\psi_{\lambda}(z) \bar{\psi}_{\lambda}\left(z^{\prime}\right)}{\lambda}
$$

In momentum space $G$ is found to consist of two parts, $G=G_{\text {chiral }}+G_{\text {massive }}$. For $G_{\text {chiral }}$ one finds

$$
G_{\text {chiral }}\left(z, z^{\prime}\right) \propto \int \frac{d^{2} k}{(2 \pi)^{2}} \frac{\sigma_{1} k_{1}+\sigma_{2} k_{2}}{k_{0}^{2}-k_{1}^{2}} e^{-i\left(x-x^{\prime}\right) k_{1}-i\left(t-t^{\prime}\right) k_{2}} .
$$

This is the 2-dimensional massless propagator of a chiral fermion. The prefactor which is left out in (39) gives the $s$-dependence of the propagator. It reproduces the result we found earlier that it is bound to the wall with an exponential fall off when going away. A similar form for the propagator exhibiting its chiral structure was obtained in [52, 19]. In addition to the chiral propagator also the one for the heavy modes can be computed

$$
G_{\text {massive }}\left(z, z^{\prime}\right)=\int \frac{d^{3} k}{(2 \pi)^{3}} \frac{\sigma^{\mu} k_{\mu}+M}{k^{2}-m_{0}^{2}} e^{-i\left(z-z^{\prime}\right) k} ; \mu=1,2,3
$$

with

$$
M=\left(\begin{array}{cc}
-m(s) & m_{12}(s) \\
0 & -m\left(s^{\prime}\right)
\end{array}\right)
$$

${ }^{2}$ This is even more so for the following reason. In 49] it was pointed out that on a domain wall one should find the covariant anomaly. This is indicated by the strength of divergence of the Chern-Simons current. On the other hand, the anomaly of a 2-dimensional chiral theory is the consistent one as required by the Wess-Zumino consistency condition 50. In 51 it was shown that by adding an extra term the consistent anomaly can be made covariant. It may be expected [49] that such an extra piece can be provided in the 2-dimensional system from the effective Chern-Simons action in 3-dimensions. 
where

$$
m_{12}=\frac{k_{0}+k_{1}}{k_{2}^{2}+m_{0}^{2}}\left[m(s) m\left(s^{\prime}\right)+i k_{2}\left(m\left(s^{\prime}\right)-m(s)\right)-m_{0}^{2}\right] .
$$

This describes a massive fermion. By analytic continuation to imaginary time the propagators (39) and (40) can be written in euclidean space where they will be denoted by $S_{E}$ and can such be used for the computation of the effective action in background gauge fields $A_{\mu}$ which in 1-loop is

$$
S_{e f f}[A]=\frac{1}{2} \int d^{3} z d^{3} z^{\prime} A_{\mu}(z) V^{\mu \nu} A_{\nu}\left(z^{\prime}\right)
$$

where

$$
V^{\mu \nu}=\operatorname{Tr} \gamma^{\mu} S_{E}\left(z, z^{\prime}\right) \gamma^{\nu} S_{E}\left(z, z^{\prime}\right) .
$$

The expression in (43) can be calculated in the $m_{0} \rightarrow \infty$ limit taking the gauge fields to be slowly varying. One finds the effective action to consist of two parts

$$
S_{\text {eff }}=S_{\text {eff }}^{C S}+S_{\text {eff }}^{\text {chiral }}
$$

with a Chern-Simons piece

$$
S_{e f f}^{C S}=\epsilon^{\mu \rho \nu} \frac{-i}{8 \pi} \int d^{3} z \operatorname{sign}(s) A_{\mu} \partial_{\rho} A_{\nu}
$$

with $\mu, \nu, \rho=1,2,3$ and a chiral piece

$$
S_{\text {eff }}^{\text {chiral }}=-\frac{m_{0}^{2}}{32 \pi^{2}} \int d^{3} z d^{3} z^{\prime} A_{a}(z) A_{b}\left(z^{\prime}\right) \frac{y^{a} y^{* b}+y^{b} y^{* a}}{\|y\|^{4}}\left[1-f\left(y, s-s^{\prime}\right)\right]^{2} \operatorname{sech}^{2}\left(m_{0} s\right) \operatorname{sech}^{2}\left(m_{0} s^{\prime}\right)
$$

where $y=\left(x-x^{\prime}, t-t^{\prime}\right), y^{a *}=i \epsilon_{b}^{a} y_{b}$ is the dual of $y^{a}$, the 2-dimensional index $a=x, t$ and

$$
f\left(y, s-s^{\prime}\right)=\frac{e^{-m_{0}\|z\|}}{\|z\|}\left[\|z\| \cosh \left(m_{0}\left(s-s^{\prime}\right)\right)+\left(s-s^{\prime}\right) \sinh \left(m_{0}\left(s-s^{\prime}\right)\right)\right] .
$$

The Chern-Simons part $S_{\text {eff }}^{C S}$ is the known result [15. We see, however, that there appears an additional piece $S_{\text {eff }}^{c h i r a l}$ which has not been computed before. As we will see now this piece is the extra part as advocated in [49, 51] to find the covariant anomaly on the domain wall. We compute the currents by a gauge variation

$$
\begin{gathered}
J_{\mu}=\frac{\delta S_{\text {eff }}}{\delta A_{\mu}} \\
J_{\mu}=-\frac{i}{4 \pi} \operatorname{sign}(s) \epsilon_{\mu \rho \nu} \partial^{\rho} A^{\mu}+\frac{i}{4 \pi} \delta_{\mu a} \epsilon_{a b} A^{b} \delta(s) .
\end{gathered}
$$

From the current in (50) we can see that we have obtained the Chern-Simons current as the first term. Its divergence comes from the different sign of the mass on the two sides of the wall. There is an additional piece in the current, residing exactly on the wall. It is this piece that gives an extra contribution to the divergence equations, making the anomaly on the wall the covariant one. 
(ii) We now proceed, discussing the second criticism of the Callan-Harvey computation, the regularization dependence of the Chern-Simons coefficient. For this purpose we describe a computation of the Chern-Simons current imposing a lattice regularization. We will work on an infinite lattice and keep the odd dimensions $d=3$, although the results hold for arbitrary odd dimensions. We are interested in the low energy coefficient $c$ of the Chern-Simons action $\Gamma_{C S}$ which is obtained when the heavy fermions are integrated out. This leads to an effective action $S_{e f f}=c \Gamma_{C S}$,

$$
\Gamma_{C S}=\epsilon_{\mu \nu \rho} \int d^{3} x A_{\mu} \partial_{\nu} A_{\rho} .
$$

The coefficient $c$ is dimensionless and the Chern-Simons operator will therefore not decouple for large fermion masses. A discussion that heavy fermion masses may not really decouple from the low energy physics is given in [53]. The Chern-Simons coefficient $c$ can be computed from the low energy portion of the vacuum polarization graph in fig.4. One obtains

$$
c=\left.\frac{i}{3 !} \epsilon_{\mu \nu \rho} \frac{\partial}{\partial q_{\nu}} \int \frac{d^{3} p}{(2 \pi)^{3}} \operatorname{Tr}\left[S(p) \Lambda_{\mu}(p, p-q) S(p-q) \Lambda_{\rho}(p-q, p)\right]\right|_{q=0} .
$$

Here $S(p)$ is the free fermion lattice propagator which will be specified later and $\Lambda_{\mu}$ is the photon vertex. The integration in (52) is understood to be taken over the 3 -dimensional Brillouin zone. A first observation is that due to gauge invariance the photon vertex may be replaced in favour of the fermion propagator via the Ward identity

$$
\Lambda_{\mu}(p, p)=-i \frac{\partial}{\partial p_{\mu}} S^{-1}(p) .
$$

Upon differentiation with respect to $\partial / \partial q_{\rho}$, the coefficient can be written as

$$
c=\frac{-i}{3 !} \epsilon_{\mu \nu \rho} \int \frac{d^{3} p}{(2 \pi)^{3}} \operatorname{Tr}\left\{\left[S(p) \partial_{\mu} S^{-1}(p)\right]\left[S(p) \partial_{\nu} S^{-1}(p)\right]\left[S(p) \partial_{\rho} S^{-1}(p)\right]\right\} .
$$

The free lattice propagators $S$ contains the lattice momenta $\sin (p)$. Therefore the lattice integral (54) appears to be quite horrible to compute. By exploiting its topological properties its calculation will become tractable, however. The topological significance of the above integral can be seen by noting that $S^{-1}$ may be generically written as

$$
\begin{aligned}
S^{-1} & =a(p)+i \vec{b}(p) \vec{\sigma} \\
& =N(p)[\cos (|\theta(p)|)+i \hat{\theta} \vec{\sigma} \sin (|\theta(p)|)] \\
& \equiv N(p) V(p)
\end{aligned}
$$

where

$$
N(p)=\sqrt{a^{2}+\vec{b}(p) \vec{b}(p)}, \quad \vec{\theta}(p)=\hat{b} \arctan (|\vec{b}| / a) .
$$

Quantities with an arrow denote a 3-dimensional vector and with a hat the corresponding unit vector. In this notation $V(p)$ is seen to be a $2 \times 2$ unitary matrix. The integral (54) does not depend on $N(p)$ provided that $S^{-1}(p)$ does not vanish. Thus $S$ and $S^{-1}$ may be replaced 


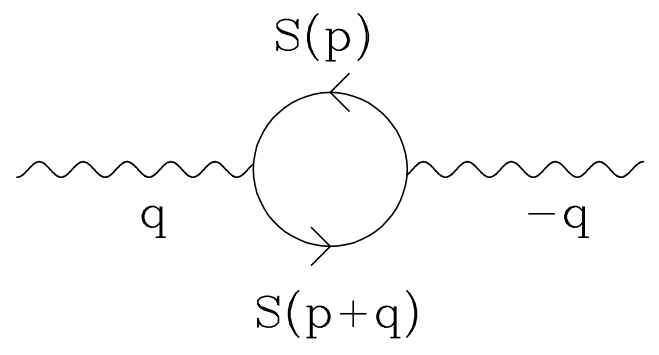

Figure 4: The vacuum polarization graph. The low energy portion of this graph leads to the Chern-Simons coefficient to be calculated for the anomaly.

everywhere by $V$ and $V^{\dagger}$. The matrix $V$ describes a mapping from the torus $T^{3}$ to the sphere $S^{3}$. The integral (54) is then nothing else but the winding number of this map. Consequently it can only take integer values up to some normalization constant. The 3-dimensional example we have worked out here can be extended to arbitrary dimensions.

To be specific we now take the usual fermion propagator for Wilson fermions for a constant mass $m$,

$$
S^{-1}(p)=\sum_{\mu=1}^{3} i \sigma_{\mu} \sin p_{\mu}+m+r \sum_{\mu=1}^{3}\left(1-\cos p_{\mu}\right) .
$$

The winding number can only change where $S^{-1}=0$. This happens only for momenta at the corners of the Brillouin zone and when the ratio $m / r=0,2,4,6$ as can be seen from (8). For the ratio $m / r \rightarrow \pm \infty, V(p) \rightarrow \pm 1$ and the integral vanishes. Therefore the ChernSimons coefficient will be zero for $m / r<0$ and $m / r>6$. Otherwise the integral (54) is piecewise constant and gives only contributions for momenta in an infinitesimal region around the Brillouin corners at the given values of $m / r$. To evaluate the integral we then only have to pick up these contributions and sum over them. Since the change of the winding number happens at the corners of the Brillouin zone we may expand the sin and cos function which leads us to evaluate

$$
\begin{aligned}
\frac{d c}{d m} & =-i \sum_{k=0}^{3}(-1)^{k} \frac{d}{d m} \int \frac{d^{3} p}{(2 \pi)^{3}} \frac{m-2 r k}{\left[p^{2}+(m-2 r k)^{2}\right]^{2}} \\
& =-\frac{i}{2 \pi} \sum_{k=0}^{3}(-1)^{k}\left(\begin{array}{l}
3 \\
k
\end{array}\right) \delta(m-2 r k) .
\end{aligned}
$$

Our final task is then a trivial integration to get the coefficient as

$$
c=-\frac{i}{4 \pi} \sum_{k=0}^{3}\left(\begin{array}{l}
3 \\
k
\end{array}\right) \frac{m-2 r k}{|m-2 r k|} \text {. }
$$


We want to remark at this point that the above derivation can be extended for arbitrary dimensions. The integral (54) describes then a map from the torus $T^{d}$ onto the sphere $S^{d}$ and the homotopy classes of these mappings are identified by integers. Therefore the whole calculation of the Chern-Simons coefficient follows very closely the above discussion.

We now want to apply the result found above for the domain wall model we are interested in. We plot in fig.5 the integer part of the integral (54) as a function of $m / r$ (dotted line). The dashed line corresponds to the chiral zeromode spectrum as discussed in section (2.1). A value of +1 means one chiral fermion with positive chirality, -2 means two chiral fermions with negative chirality etc. Clearly the Chern-Simons coefficient follows exactly the behaviour of the change of the chiral zeromode spectrum with $m / r$.

One peculiar feature of the Chern-Simons coefficient is that it is zero for negative $m / r$. Since in the domain wall model $r$ is positive and $m$ has different signs on the two sides of the wall, this means that the Chern-Simons current flows only on one side of the wall. This is to be contrasted with the continuum analysis which reveals that the current flows with equal strength but opposite signs on the two sides of the wall. Furthermore, if we take for example $m=r=1$ the value of the Chern-Simons coefficient is $c=-i / 2 \pi$ for $m>0$ which is exactly twice the continuum value. Thus we find that the strength of the Chern-Simons current depends on the regularization used as already emphasized in [47]. Of course, the divergence of the Chern-Simons current across the wall comes out the same in both calculations giving the correct strength of the anomaly. It is quite remarkable that the appearance of the anomaly on the lattice holds also for values of the domain wall mass at the order of the lattice cut-off. The fact that the Chern-Simons current flows only on one side of the wall motivates even more Shamir's approach to use free boundary conditions with the signs of the Wilson coupling and the mass chosen such that we will have current flow.

The result for the 3-dimensional system discussed above can be generalized to arbitrary dimensions. In $d=2 n+1$ dimensions the number of chiral zeromodes bound to the domain wall is for $2 k<\left|\frac{m}{r}\right|<2 k+2,0 \leq k \leq d-1$

$$
\left(\begin{array}{c}
d-1 \\
k
\end{array}\right)
$$

with the chirality of the modes to be $(-1)^{k} \operatorname{sign}(m)$. The corresponding Chern-Simons current is

$$
\frac{J_{\mu}^{C S}(\text { lattice })}{J_{\mu}^{C S}(\text { continuum })}=2(-1)^{k}\left(\begin{array}{c}
d-1 \\
k
\end{array}\right)
$$

with the continuum Chern-Simons current in $d=2 n+1$ dimensions given as

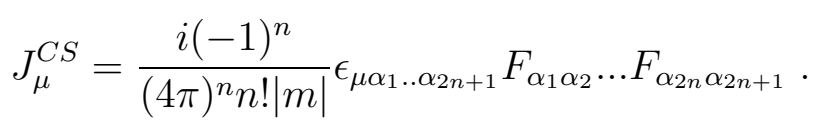

The above scenario can be implemented on a finite lattice of size $L^{2} L_{s}$. The euclidean lattice 


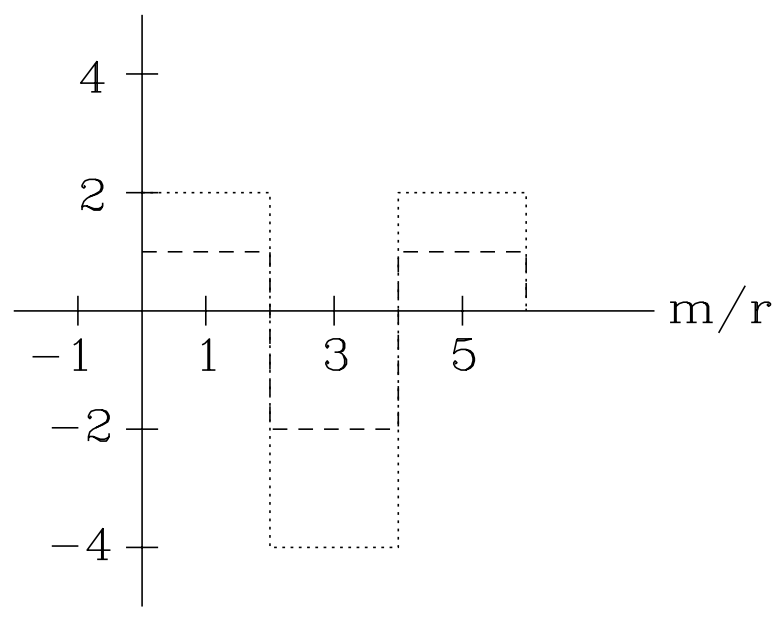

Figure 5: The integer part of the Chern-Simons coefficient (dotted line) as a function of $m / r$. The dashed line indicates the chiral zeromode spectrum. +1 means 1 chiral zeromode with positive chirality, -2 is two chiral zeromodes with negative chirality. For $m / r<0$ and $m / r>2 d$ the spectrum contains no chiral zeromode and the Chern-Simons current vanishes, accordingly.

action for free domain wall fermions is given by

$$
\begin{aligned}
S & =\frac{1}{2} \sum_{z, \mu}\left[\bar{\psi}_{z} \gamma_{\mu} \psi_{z+\mu}-\bar{\psi}_{z+\mu} \gamma_{\mu} \psi_{z}\right]+m(s) \sum_{z} \bar{\psi}_{z} \psi_{z} \\
& +\frac{r}{2} \sum_{z, \mu}\left[-2 \bar{\psi}_{z} \psi_{z}+\bar{\psi}_{z} \psi_{z+\mu}+\bar{\psi}_{z+\mu} \psi_{z}\right]
\end{aligned}
$$

with $z=(x, t, s)$ and $\mu=1, \ldots, 3$. We can couple external abelian gauge fields to the fermions in close analogy to the Hamiltonian (31) by making the finite lattice differences gauge covariant, see eqs. (29,30). Note that at this point the gauge fields are purely external. How to write down a dynamical gauge field action will be discussed in the next section. The lattice current is obtained by a gauge variation of the action and reads [1]

$$
j_{z}^{\mu}=\frac{1}{2}\left[\bar{\psi}_{z} \gamma_{\mu} U_{z, \mu} \psi_{z+\mu}+\bar{\psi}_{z+\mu} \gamma_{\mu} U_{z, \mu}^{*} \psi_{z}\right]+\frac{r}{2}\left[\bar{\psi}_{z} U_{z, \mu} \psi_{z+\mu}-\bar{\psi}_{z+\mu} U_{z, \mu}^{*} \psi_{z}\right] .
$$

We will choose a t-dependent external gauge field

$$
U_{z, \mu=2}=\exp \left\{-i q\left[\frac{L}{2 \pi} E_{0} \cos \left(\frac{2 \pi}{L}(t-1)\right)\right]\right\}
$$

and make $E_{0} \ll 1$ in order to stay in the low energy regime and below the critical momentum $k_{c}$. The $U$ 's in the other directions have been set to one.

As we are considering free fermions in an external gauge field background, the matrix elements of the current can be computed from the inverse fermion matrix using standard numerical techniques like Conjugate Gradient for the matrix inversion. Note, that in this way 
the computation of the current is -up to rounding errors- exact. In particular, no simulation is involved.

Since the width of the wavefunction in the extra dimension is finite, see fig.1, we will sum the current over the range in s corresponding to the support $\Lambda_{\psi}$ of the wavefunction. Then we evaluate the divergence

$$
<\partial_{i} j_{i}>\equiv \sum_{s \in \Lambda_{\psi}} \partial_{i} j_{i}(t, x, s), i=(t, x)
$$

According to the discussion for the Hamiltonian formalism we expect the anomaly equation to be satisfied

$$
<\partial_{i} j_{i}>= \pm \frac{q^{2}}{2 \pi} E_{e f f}(t)
$$

where the effective electric field $E_{\text {eff }}$ for small $E_{0}$ is given by

$$
E_{e f f}=\frac{\sin \left(\frac{2 \pi}{L}\right)}{\frac{2 \pi}{L}} E_{0} \sin (t-1)
$$

and the sign is determined by the chirality of the mode. Choosing first the Wilson parameter $r=0$ and a charge of $q=+1$, it is found that the divergence of the current (66) vanishes. This is perfectly consistent with the fact that for $r=0$ the doubler modes are still in the spectrum, cancelling the anomaly.

Turning the Wilson parameter on should change the picture. We expect the doublers to become decoupled and the anomaly equation to be satisfied. The divergences of the currents computed for charges of $q=3,4,5$ and chirality $+1,+1,-1$ are shown in fig.6. Each of them follow the anomaly equation individually up to a few $\%$. Taking the sum of them the total divergence vanishes which corresponds to the anomaly cancellation as predicted by the Pythagorean relation $3^{2}+4^{2}-5^{2}=0$. In particular, the numerical results confirm that the Chern-Simons current flows only on side of the wall [54]. Thus we see both, the individual anomaly for a fermion with a given charge and the cancellation of the anomalies if the fermions are in an anomaly free representation.

\section{Coupling to gauge fields}

Despite all the positive results we found in the previous sections the crucial question remains, whether we can keep the chiral zeromodes decoupled from each other when the gauge fields are made dynamical. The danger is that the gauge fields might induce an interaction between the two domain walls. We will discuss two proposals of coupling gauge fields to domain wall fermions. In a first attempt, the gauge field is 5-dimensional. The gauge couplings are chosen differently for the 4-dimensional fields and in the fifth direction. The hope in this approach is that by tuning the 4-dimensional gauge coupling to zero and making the coupling in the fifth direction strong at the same time, physics is confined to 4 dimensions. One then might perform the usual continuum limit in 4 dimensions while keeping the chiral structure of the theory. 


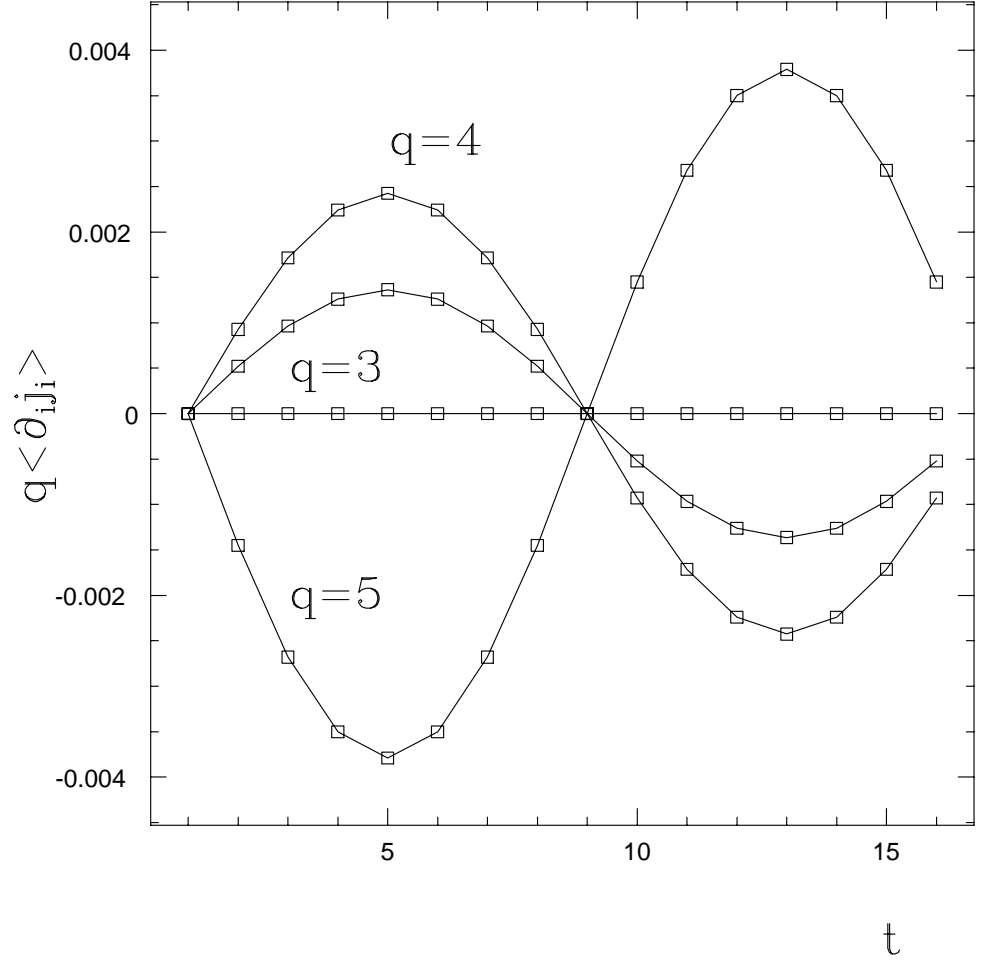

Figure 6: The divergence of the $(1+1)$-dimensional gauge current in an external gauge field. The system consists of fermions with charge $q=3,4,5$ and chirality,,++- , respectively. Each of the currents obey the lattice anomaly equation individually. The sum of the currents vanish due to the anomaly cancellation.

We will see, however, that this path does not lead to the desired result, namely a 4dimensional chiral gauge theory. The failure of this approach suggests that one has to keep the gauge fields strictly 4-dimensional from the very beginning. In order not to couple both walls, the gauge fields have to be switched off on one of the domain walls such that only one wall is gauged. We will thus have a situation with identical 4-dimensional gauge fields on a number of $s$-slices around a wall and $U=1$ in the complementary region around the anti-wall. Clearly, at the boundary where both regions meet we loose gauge invariance. This can be repaired by introducing a scalar (or Stückelberg) field at this boundary. However, as we will see, there exist light mirror fermions living on the boundary. They can interact with the chiral zeromode on the gauged domain wall thus rendering the theory vectorlike again.

\subsection{Dimensional reduction}

In this first way to couple the gauge fields the gauge interaction is split into a purely 4dimensional part and a piece that contains the gauge fields in the fifth direction. Both parts are equipped with different gauge couplings. For the possible phase structure and the question of whether one may obtain a chiral gauge theory we will consider a 5-dimensional system. The reason for making a departure from our 3-dimensional setup will become clear later. For the discussion it is sufficient in a first step to consider the pure gauge theory alone. We will choose 
U(1)-gauge fields. The action becomes

$$
S(U)=\sum_{x, s}\left(\beta_{4} \sum_{\mu, \nu=1}^{4} \operatorname{Re}\left(U_{x, \mu}^{s} U_{x+\mu, \nu}^{s} U_{x+\nu, \mu}^{* s} U_{x, \nu}^{* s}\right)+\beta_{5} \sum_{\mu} \operatorname{Re}\left(U_{x, \mu}^{s} V_{x+\mu}^{s} U_{x, \mu}^{* s+1} V_{x}^{* s}\right)\right) .
$$

Here $\beta_{4}=1 / g_{4}^{2}$ is the 4-dimensional and $\beta_{5}=1 / g_{5}^{2}$ the 5 -dimensional inverse gauge coupling squared. We have denoted with $U$ the gauge fields living in 4 dimensions and with $V$ the ones in the extra dimension. Thinking of $s$ more as a flavour space than an extra dimension, we have denoted the $s$-dependence with a superscript on the fields. Note that the role of the $V$-fields look very much as that of scalar fields coupling to 4-dimensional gauge fields in some peculiar flavour space. We are interested in the properties of this 5-dimensional gauge theory in the limit of large $\beta_{4}$ corresponding to the continuum limit in 4 dimensions.

Letting the gauge fields be $U_{x, \mu}=e^{i \theta_{x, \mu}}$ and $V=e^{i \theta_{x, \mu}^{\prime}}$ the path integral is

$$
Z=\int \mathcal{D} \theta_{x, \mu} \mathcal{D} \theta_{x, \mu}^{\prime} e^{-S\left(\theta_{x, \mu}, \theta_{x, \mu}^{\prime}\right)} .
$$

We consider this model in the meanfield approximation [55, 56] which is obtained by inserting

$$
1=\int \mathcal{D} v_{x, \mu} \int_{-i \infty}^{i \infty} \mathcal{D} \alpha \exp \left\{\sum_{x, \mu}\left[\alpha_{x, \mu}\left(v_{x, \mu}-\theta_{x, \mu}\right)\right]\right\}
$$

in the path integral. In ([1) the mean field $v$ is a complex variable living on the links of the lattice and $\alpha$ an auxiliary field. There is an analogous expression for $\theta^{\prime}$. Now the integration over the original link variables $\theta$ and $\theta^{\prime}$ decouple and the path integral becomes

$$
Z=\int \mathcal{D} v \int \mathcal{D} \alpha \exp \left\{\sum_{x, s}\left(\beta_{4} \sum_{\mu, \nu=1}^{4} \operatorname{Re} v_{x, \mu \nu}+\beta_{5} \sum_{\mu} \operatorname{Re} v_{x, \mu 5}\right)+W(\alpha)+W\left(\alpha^{\prime}\right)-v \alpha-v^{\prime} \alpha^{\prime}\right\}
$$

where

$$
W(\alpha)=\int_{0}^{2 \pi} d \theta_{x, \mu} e^{i \alpha \theta_{x, \mu}}
$$

and $v_{x, \mu \nu}$ denotes the product of the $v$ 's around an elementary plaquette in 4 dimensions and $v_{x, \mu 5}$ the corresponding expression in the fifth direction. A saddle point of the action in (72) is obtained by solving

$$
\begin{aligned}
& \frac{\partial S}{\partial v}=\alpha ; \quad \frac{\partial S}{\partial v^{\prime}}=\alpha^{\prime} \\
& \frac{\partial W}{\partial \alpha}=v ; \quad \frac{\partial W}{\partial \alpha^{\prime}}=v^{\prime} .
\end{aligned}
$$

Searching for translational invariant solutions by setting $v$ and $v^{\prime}$ to constants, the explicit equations at tree level are 56]

$$
\begin{aligned}
\alpha & =2(d-2) \beta_{4} v^{3}+2 \beta_{5} v^{\prime 2} v+2 \beta_{4} v \\
\alpha^{\prime} & =\beta_{5}\left(2(d-1) v^{2} v^{\prime}+2 v^{\prime}\right)
\end{aligned}
$$

and

$$
v=\frac{I_{1}(\alpha)}{I_{0}(\alpha)} ; \quad v^{\prime}=\frac{I_{1}\left(\alpha^{\prime}\right)}{I_{0}\left(\alpha^{\prime}\right)}
$$




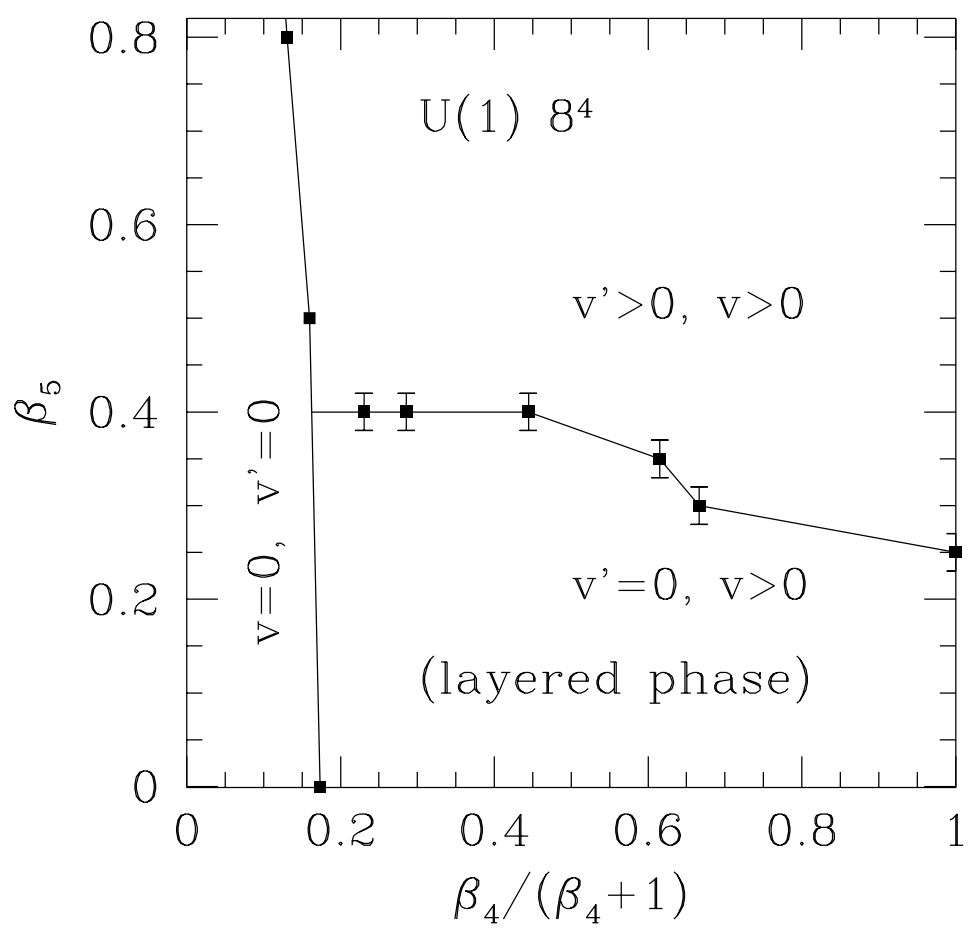

Figure 7: The phase diagram as obtained from a numerical simulation of the 5-dimensional $\mathrm{U}(1)$ gauge theory for an $8^{4}$ lattice. Only the portion for large $\beta_{4}$ is relevant for the continuum limit.

where

$$
I_{k}(\alpha)=\frac{1}{\pi} \int_{0}^{\pi} d \theta \cos ^{k}(\theta) e^{\alpha \cos (\theta)} .
$$

In principle there appear three different phases see [56] and fig.7. However, we are only interested in the large $\beta_{4}$ limit and will not discuss the phase with $v=0$ and $v^{\prime}=0$. For large $\beta_{4}$ one finds a layered phase with $v>0, v^{\prime}=0$ and separated from it by a phase transition at $\beta_{5}^{c}$ a symmetry broken phase with $v>0$ and $v^{\prime}>0$. The layered phase is characterized by the fact that a charged particle will only move along the layer and can not hop between layers. Therefore if one would live inside a layer one would basically only experience 4-dimensional physics. In [56] it was argued that such a phase only appears when the lower dimension is greater than two. Thus an investigation of a 3-dimensional system would simply miss the layered phase and might lead to wrong conclusions. In fig. 7 we show the phase diagram of the 5 -dimensional pure $\mathrm{U}(1)$ gauge theory. It is obtained by means of numerical simulations on an $8^{4}$ lattice. As is predicted by the meanfield computation, there are indeed two phases at large $\beta_{4}$. One of them has $v>0$ ans $v^{\prime}>0$ and corresponds to the symmetry broken phase. For $\beta_{5}<\beta_{5}^{c}$ there appears the layered phase which is the promising phase to obtain 4-dimensional physics. Taking the 1-loop corrections to the mean field equations into account, the phase diagram remains stable.

The fermions are included via the action

$$
S_{F}=\sum_{x, s} \sum_{\mu=1}^{4} \bar{\psi}_{x}^{s}\left[\left(\partial_{x}(U) \gamma_{\mu}-\Delta_{x}(U)+m(s)\right] \psi_{x}^{s}\right.
$$




$$
+\frac{1}{2} \sum_{x, s} \bar{\psi}_{x}^{s}\left[V_{x}^{s} \delta_{s, s+\mu_{5}}\left(1+\gamma_{5}\right)+V_{x}^{* s} \delta_{s-\mu_{5}, s}\left(1-\gamma_{5}\right)-2 \delta s, s\right] \psi_{x}^{s}
$$

where we have separated the 4-dimensional part from the part in the fifth direction and set $r=1$. Again we have written the $s$-label as a "flavour" label and $\mu_{5}$ denotes a unit vector in the 5-direction. In [24] it was shown that the fermions lead only to a slight modification of the mean field equations and that the phase structure depicted in fig.7 remains stable when fermions are taken into account. The interesting question, of course, concerns the dynamics of the fermions in the layered phase. One might get a hint by an inspection of the action itself [25]. In the layered phase $v^{\prime}=0$ corresponding to set $V$ in the action (78) to zero. But then the action looks like a normal 4-dimensional Wilson action in each layer. Indeed, in [24] the fluctuations to the tree level saddle point solution were computed to get the fermion propagator. As a result one finds for every $s$-slice the 4-dimensional Wilson propagator. This means that the theory -though truly 4-dimensional- is vectorlike in each s-layer. Therefore we have to throw away the region $\beta_{4} \gg 1$ and $\beta_{5}<\beta_{5}^{c}$ as a possible corner of the phase diagram for a construction of a chiral gauge theory from domain wall fermions.

In [27] it could be proven by the hopping parameter expansion that for $\beta_{5} \ll 1$ the fermion propagator is parity invariant, with the parity transformation defined such that for $x_{i} \rightarrow-x_{i}$ with $x$ being the 4-dimensional coordinate

$$
\psi \rightarrow \gamma_{0} \psi, \bar{\psi} \rightarrow \bar{\psi} \gamma_{0}
$$

Therefore there is a complete symmetry between left and right handed modes. Thus the theory is completely vectorlike in each layer strengthening the above conclusions.

What remains is the possibility that $\beta_{5}>\beta_{5}^{c}$ [25], i.e. $v>0, v^{\prime}>0$. In this situation we have symmetry breaking. In each s slice there is a $G=U(1) \otimes U(1)$ symmetry such that the total symmetry group is $G^{L_{s}}$ when the extent of the system in the fifth direction is $L_{s}$. For $v^{\prime}>0$ this symmetry is broken to its diagonal subgroup $G$. Then we are left with $L_{s}-1$ massive gauge bosons with a mass $m_{G} \propto v^{\prime}$. However, there will remain one massless gaugefield which does not depend on $s$. This gauge field couples equally to the modes at the domain and the anti-domain wall. Therefore, although we still would have the zeromodes on the domain walls, they can communicate via the massless gauge boson. We expect therefore the model again to be vectorlike.

\subsection{Waveguide model}

The previous section showed that starting with 5-dimensional gauge fields does - most probablynot lead to a chiral theory via dimensional reduction. Obviously the gauge fields have to be strictly 4-dimensional from the very beginning. This becomes a more natural point of view if one considers the extra dimension as a flavour space with a somewhat unusual flavour matrix. On the finite lattice with two domain walls not all s-slices (flavours) can be gauged. This would 
immediately lead to the possibility that the zeromodes on both domain walls communicate, rendering the theory vectorlike.

One might therefore try a scenario where only one of the domain walls is gauged and can interact with the gauge fields. This corresponds to taking a number of s-slices around a domain wall and put identical 4-dimensional gauge fields on each of these slices leaving the complementary $s$-slices ungauged. That the gauge fields have to be identical is again motivated from the flavour space picture. In this way both walls are completely shielded from each other. However, following the above prescription one notices that at the s-layer where a gauged s-slice meets an ungauged layer gauge invariance is broken.

One possibility is that one does not worry about broken gauge invariance [57]. Indeed, several chiral fermion proposals exist that start with broken gauge invariance [11]. It is then hoped that gauge invariance is restored in the continuum limit. However, we want to follow a path where gauge invariance is kept. Its restoration is actually very easy. One just has to remember how the Yukawa-coupling in the Standard Model is made gauge invariant, namely by introducing scalar (or Stückelberg) fields. From this point of view, the model with broken gauge invariance can be thought of as the gauge invariant model in the unitary gauge. Thus, both descriptions are actually completely equivalent [4]. The proposal to couple gauge fields in the domain wall model is

- keep the gauge fields strictly 4-dimensional,

- gauge only a number of s-slices around one domain wall,

- introduce scalar fields at the boundary of the gauged region.

The gauge fields are then confined to a region in $s$ between the boundaries where the scalar fields live. Thinking of electromagnetism this resembles a situation where the electromagnetic waves are trapped in a waveguide which suggests the name "waveguide model" that we will use from now on.

The introduction of the scalar fields gives rise to a coupling between the fermions and these fields. It is then natural to equip the resulting interaction with a Yukawa-coupling $y$. To get a feeling for the physics of the model proposed, one could first switch off the Yukawa coupling and set the gauge fields to one. In this situation the zeromode spectrum can be obtained again from the Hamilton formalism. The result for a 3-dimensional system is shown in fig.8a.

The situation resembles a combination of the domain wall model and the boundary fermion model. Setting $y=0$ cuts the waveguide region completely off and produces open boundary conditions. Due to our discussion in section (2.2) we expect to find chiral zeromodes where the mass term is negative. This is indeed what happens. We find the original chiral zeromode on the domain wall $\psi_{L}$ and a chiral surface mode -denoted in the following as "mirror fermion"with opposite chirality $\chi_{R}$ at the boundary. A complementary picture is obtained in the s-region outside the waveguide. 


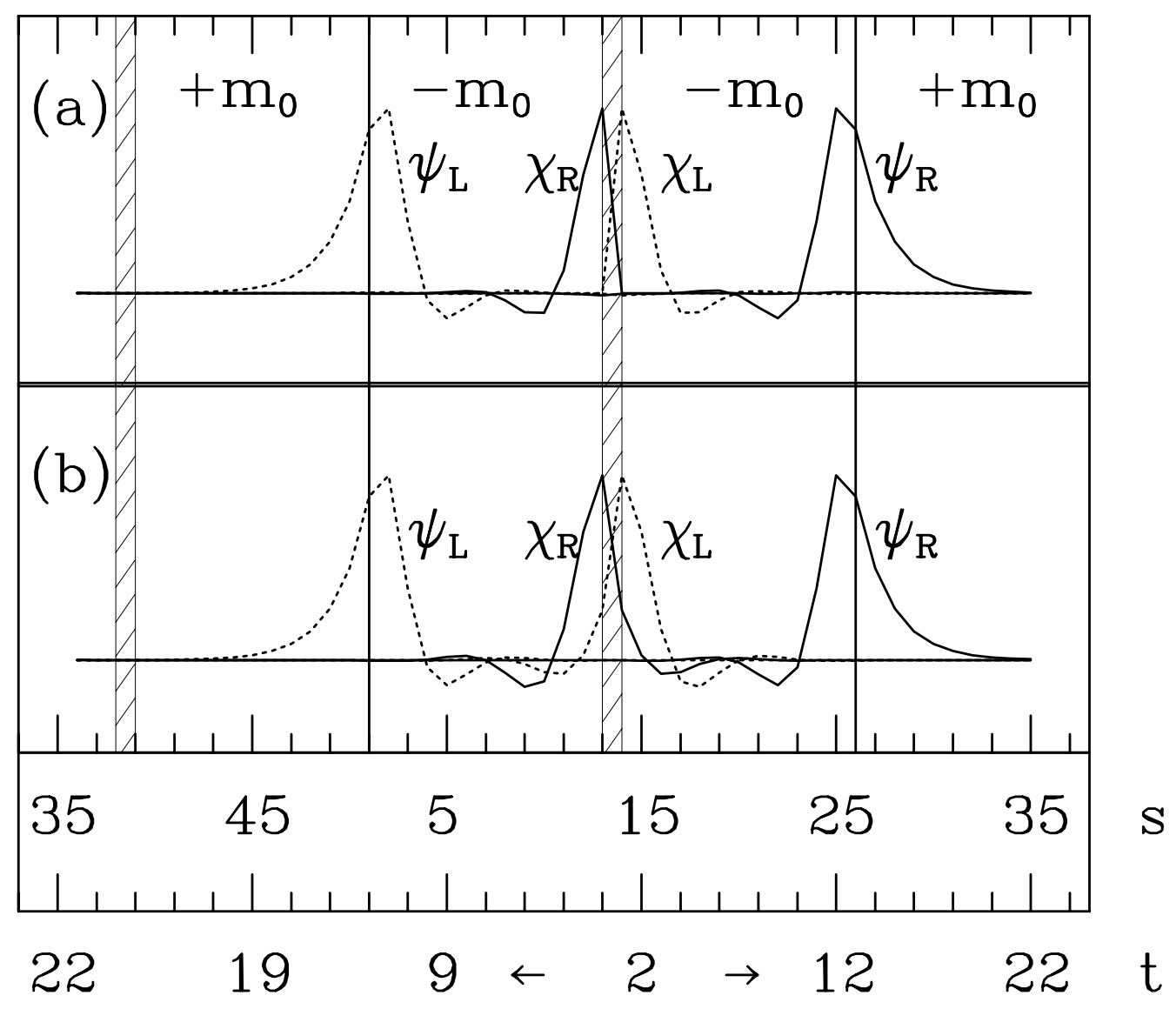

Figure 8: The zeromode spectrum in the waveguide model. The boundary where the scalar fields live are indicated by the shaded stripes. The position of the domain walls are given by the vertical bar. Fig.8a corresponds to $y=0$ and fig.8b to $y>0$. 
Staying in a meanfield setup by giving the scalar field a vacuum expectation value $v$, we show in fig.8b the spectrum for a non-zero value of $y$. Now the mirror mode can leak out of the waveguide region and can combine with the mirror mode from outside the waveguide region to form a Dirac particle. The mass of this particle is expected to follow the usual perturbative relation $m_{f}=y v$.

Let us make clear what we would like to achieve with the waveguide model. Clearly one wants the chiral zeromode on the domain wall to survive. At the same time, the mirror modes at the waveguide boundary should be heavy and therefore decouple from the spectrum. This scenario looks hopeless if the mirror fermion mass follows the perturbative relation. Approaching the phase transition to the symmetric phase, $v$ (in lattice units) approaches zero. Then also the mirror fermion becomes massless and can consequently not be decoupled. Moreover, in the symmetric phase the mirror fermions are expected to be massless since there the vacuum expectation value vanishes identically. We would therefore end up with a variant of Montvay's mirror fermion model [58].

What gives hope for the waveguide model is its resemblance to Yukawa-models on the lattice. In fact, concentrating only on the waveguide boundary one might consider the model as being just of the Yukawa-type. Such models have been investigated intensively in the last years. The most surprising and unexpected outcome of these investigations was the discovery of a new phase at large, non-perturbative values of the Yukawa-coupling [32, 33.

When the Yukawa-coupling is very large, the fermions can easily combine with the scalar fields and form massive bound states even in the symmetric phase where the vacuum expectation value is zero. The masses of these states can be at the order of the cutoff such that they decouple in the continuum limit. What made the Yukawa models not successful for a regularization of a chiral gauge theory was that the continuum limit in the strong coupling region did not resemble the Standard Model at all. The fermion spectrum in the continuum limit consisted of only a neutral Dirac fermion. The necessary charged fermion that would couple to the left handed gauge field is simply missing and does not appear in the spectrum. What is important is, that on the other hand it was easy to give the neutral fermion a large mass.

For the domain wall model the situation is different in that we still would have the chiral zeromode on the domain wall. Here the strong coupling phase would just serve to make the modes at the waveguide boundary heavy. The real physics - and hopefully a chiral theorywould appear along the domain wall. The main question in the domain wall approach to chiral fermions on the lattice is then whether in the waveguide realization a strong coupling phase exists.

This makes it necessary to explore the phase diagram of the waveguide model. In doing so we will impose some simplifications. First, we set the gauge fields $U=1$. This is certainly justified as in the continuum limit the gauge coupling becomes small and the gauge fields might be treated perturbatively. Furthermore, the investigations of Yukawa models on the lattice revealed the strong coupling region leaving out the gauge fields, too. Therefore it appears 


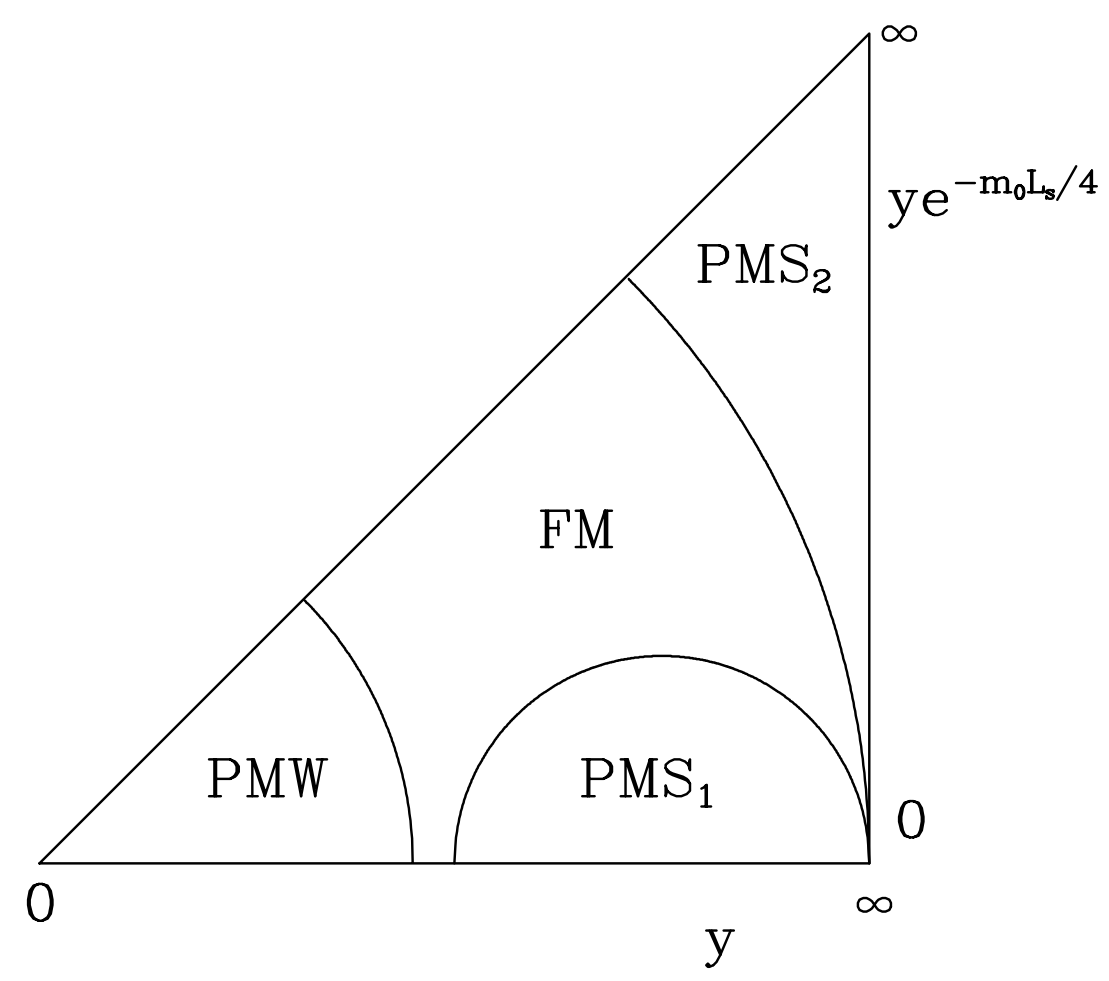

Figure 9: The phase diagram which would make the waveguide model successful.

to be sufficient to study the system with fermions and scalar fields alone. Secondly, we will go back again to a 3-dimensional system for the numerical simulations that will be presented below. Experience from earlier work suggests that they resemble 4-dimensional systems quite closely [59]. In particular the strong coupling behaviour could also be identified in this lower dimensional models. Thus the appearance or non-appearance of a strong coupling region in the 3-dimensional model would strongly point towards that such a region may or may not exist also in higher dimensional models.

A sketch of the phase diagram which would make the waveguide model successful is shown in fig.9. There are two Yukawa-couplings. First there is the coupling we introduced at the waveguide boundary to couple the scalar fields directly to the fermions. Secondly, due to the exponential fall off, the domain wall fermion will have an -exponentially suppressed-overlap with the modes at the waveguide boundary and hence couples to the scalar field with a strength $y \exp \left(-m_{0} L_{s} / 4\right)$. (The distance of the waveguide boundary to the domain wall is $L_{s} / 4$, see fig.8). The phase diagram in these two couplings may have the following phases.

- FM In the ferromagnetic phase the symmetry is spontaneously broken and the scalar field assumes a vacuum expectation value $v>0$. If the model would be successful, there should be two regions in the FM phase. One with weak coupling behaviour $m_{F} \approx y v$ and a strong coupling region with heavy fermions.

- PMW This is the weak paramagnetic phase. Here the symmetry is restored and the vacuum expectation value $v=0$. In this phase the mirror fermions living at the waveguide 
boundary are massless and do not decouple from the spectrum. Therefore it is not possible to construct a chiral gauge theory in this phase.

- $\mathbf{P M S}_{1}$ A strong paramagnetic phase. This is the phase we are looking for. Here due to the large Yukawa-coupling the binding of the fermions to the scalar fields at the waveguide boundary becomes so strong that they combine and form massive bound states. At the same time the chiral zeromode on the domain wall remains massless. Therefore the mirror fermions are expected to decouple and we are -hopefully- left with a chiral gauge theory on the domain wall.

- $\mathbf{P M S}_{2}$ Again a strong paramagnetic phase. However, this time the extension of the system in the extra dimension is so short that the overlap of the chiral zeromode on the domain wall with the waveguide boundary becomes non-negligible. Therefore the domain wall modes can also combine with the scalar fields getting massive in this way. Clearly also in this phase no chiral fermions can be obtained.

In the following, we will use PMW to indicate a weak coupling behaviour of the fermion mass, $m_{f} \propto v$. PMS will indicate strong coupling behaviour with massive bound states. Note, that in this way PMS and PMW will not necessarily stand for weak and strong values of the Yukawa-coupling $y$.

\subsection{The action of the waveguide model}

After the qualitative discussion of the previous subsection, we want to become more concrete and will construct the lattice action suitable for eventual simulations. As we discussed above, we take the same gauge field on all $s$-slices inside the waveguide. The index for the extra dimension will be chosen as a superscript to show the resemblance to the flavour space picture. We define gauge transformations on the fermion field as

$$
\begin{gathered}
\Psi_{x}^{s} \rightarrow g_{x} \Psi_{x}^{s}, \bar{\Psi}_{x}^{s} \rightarrow \bar{\Psi}_{x}^{s} g_{x}^{\dagger} \quad s \in W G \\
\Psi_{x}^{s} \rightarrow \Psi_{x}^{s}, \quad \bar{\Psi}_{x}^{s} \rightarrow \bar{\Psi}_{x}^{s} \quad s \notin W G, \\
W G=\left\{s: s_{0} \leq s \leq s_{0}^{\prime}\right\}
\end{gathered}
$$

with $g_{x}$ in a gauge group $G$. The detailed choice of the boundaries $s_{0}$ and $s_{0}^{\prime}$ is not very important, provided they are sufficiently far from the domain wall that the zeromode is exponentially small at the waveguide boundary. For symmetry reasons we shall choose $s_{0}=\left(L_{s}+2\right) / 4+1$ and $s_{0}^{\prime}=\left(3 L_{s}+2\right) / 4$, such that the right handed mode at $s=L_{s} / 2+1$ is located at the center of the waveguide, see fig. 8. With this choice we have to take $L_{s}-2$ a multiple of four.

Having made this division into a waveguide and its exterior, we note that the model has a global $G \times G$ symmetry:

$$
\begin{aligned}
& \Psi_{x}^{s} \rightarrow g \Psi_{x}^{s}, \quad \bar{\Psi}_{x}^{s} \rightarrow \bar{\Psi}_{x}^{s} g^{\dagger}, \quad s \in W G, \\
& \Psi_{x}^{s} \rightarrow h \Psi_{x}^{s}, \quad \bar{\Psi}_{x}^{s} \rightarrow \bar{\Psi}_{x}^{s} h^{\dagger}, \quad s \notin W G .
\end{aligned}
$$


With our choice for the position of the waveguide boundary, there is a symmetry involving parity plus a reflection in the $s$-direction with respect to the plane $s=s_{0}-\frac{1}{2}=L_{s} / 4+1$,

$$
\Psi_{x}^{s} \rightarrow \gamma_{d} \Psi_{P x}^{L_{s} / 2+2-s}
$$

with $P x=\left(-x_{1}, \cdots,-x_{d-1}, x_{d}\right)$ the parity transform of $x$.

The layers, where gauge invariance is broken are now $s_{0}-1$ to $s_{0}$ and from $s_{0}^{\prime}$ to $s_{0}^{\prime}+1$. As discussed in the previous section, this will be repaired by the introduction of scalar fields (or Stückelberg field) $V$ at the boundary of the waveguide. Alternatively the scalar field $V$ might be interpreted as a component of the gauge field in the extra dimension. In this sense the waveguide model is an improved variation of the actions $(69,78)$ where the gauge fields have been made 5-dimensional. It corresponds to making the gauge coupling in the fifth dimension $\beta_{5}=\infty$ except at two s-slices, namely at $s=s_{0}$ and at $s=s_{0}^{\prime}$. We obtain the gauge invariant action (suppressing the even dimensional index $x$ )

$$
\begin{aligned}
S_{\Psi} & =\sum_{s \in W G} \bar{\Psi}^{s}\left(\not \partial(U)-\Delta(U)+m^{s}\right) \Psi^{s}+\sum_{s \notin W G} \bar{\Psi}^{s}\left(\not \partial-\Delta+m^{s}\right) \Psi^{s} \\
& -\sum_{s \neq s_{0}-1, s_{0}^{\prime}}\left[\bar{\Psi}^{s} P_{L} \Psi^{s+1}+\bar{\Psi}^{s+1} P_{R} \Psi^{s}\right]+\sum_{s} \bar{\Psi}^{s} \Psi^{s} \\
& -y\left(\bar{\Psi}^{s_{0}-1} V P_{L} \Psi^{s_{0}}+\bar{\Psi}^{s_{0}} V^{\dagger} P_{R} \Psi^{s_{0}-1}\right)-y\left(\bar{\Psi}^{s_{0}^{\prime}} V^{\dagger} P_{L} \Psi^{s_{0}^{\prime}+1}+\bar{\Psi}^{s_{0}^{\prime}+1} V P_{R} \Psi^{s_{0}^{\prime}}\right),
\end{aligned}
$$

where we have supplied the Yukawa term with a coupling constant $y$ as anticipated. Note that we take the same scalar field at both waveguide boundaries. Since we have chosen $r=1$ we have written projectors in the hopping terms in $s, P_{R(L)}=\frac{1}{2}\left(1+(-) \gamma_{5}\right)$. $\not \partial(U)=\gamma_{\mu} \partial_{x}(U)$ is the usual gauge covariant lattice Dirac operator, see (29,30). The field $V_{x} \in G$ is the scalar field, which can be thought of as a (radially frozen) Higgs field, and which transforms as

$$
V_{x} \rightarrow h V_{x} g_{x}^{\dagger}
$$

The transformation given in eq. (83) remains a symmetry if $V$ transforms as

$$
V_{x} \rightarrow V_{P x}^{\dagger}
$$

Having introduced the scalar field, it is very natural to add a kinetic and an interaction term for it. It is expected that through renormalization these terms would be generated automatically. Since we have the scalar field to be radially frozen, we choose the standard lattice form of the non-linear $\sigma$ model

$$
S_{V}=-\kappa \sum_{x, \mu} \operatorname{Tr}\left(V_{x} U_{x, \mu} V_{x+\mu}^{\dagger}+\text { h.c. }\right) .
$$

The scalar hopping parameter $\kappa$ is proportional to the bare mass squared. For the pure scalar theory we will have a broken phase for $\kappa>\kappa_{c}$ as well as a symmetric phase for $\kappa<\kappa_{c}$. 


\subsection{Mirror fermion representation of the model}

To get more insight into the mode spectrum of the model and to make the interpretation of the surface modes $\chi_{L, R}$ in fig.8 as mirror fermions more plausible, we rewrite the action as follows. Relabel the right and left handed fermion fields, $\Psi_{R, L}^{s}=P_{R, L} \Psi^{s}$ as

$$
\begin{array}{ll}
\psi_{R}^{t}=\Psi_{R}^{s_{0}-1+t}, & \psi_{L}^{t}=\Psi_{L}^{s_{0}-t}, \\
\chi_{L}^{t}=\Psi_{L}^{s_{0}-1+t}, & \chi_{R}^{t}=\Psi_{R}^{s_{0}-t},
\end{array}
$$

and the same for $\bar{\Psi}_{R, L}=\bar{\Psi} P_{L, R}$ (note the reversal of $L$ and $R$ ). The new label $t$ runs from 1 to $L_{t} \equiv L_{s} / 2$. In fig. 8 we have indicated this new labeling for the zeromode wave functions shown there. With our choice for $s_{0}, s_{0}^{\prime}$ and $L_{s}$ we can define a domain wall mass for both fields $\psi$ and $\chi$, which is a step function in $t$ satisfying,

$$
\bar{\mu}^{t}=m^{s_{0}-1+t}=m^{s_{0}-t} .
$$

With this relabeling the two domain wall zeromodes will reside in the Dirac fermion field $\psi$, whereas the waveguide boundary zeromodes will reside in $\chi$. After substituting eq. (88) into eq. (84) with $U=1$, the action turns into

$$
\begin{aligned}
S_{\psi_{\chi}} & =\sum_{t=1}^{L_{t}}\left(\bar{\psi}^{t} \not \partial \psi^{t}+\bar{\chi}^{t} \not \partial \chi^{t}+\bar{\chi}^{t}\left(-\Delta+\bar{\mu}^{t}\right) \psi^{t}+\bar{\psi}^{t}\left(-\Delta+\bar{\mu}^{t}\right) \chi^{t}\right) \\
& -\sum_{t=1}^{L_{t}-1}\left(\bar{\psi}^{t} \chi^{t+1}+\bar{\chi}^{t+1} \psi^{t}\right)+\sum_{t}\left(\bar{\chi}^{t} \psi^{t}+\bar{\psi}^{t} \chi^{t}\right) \\
& -y \bar{\chi}^{1}\left(V P_{L}+V^{\dagger} P_{R}\right) \chi^{1}-y \bar{\psi}^{L_{t}}\left(V^{\dagger} P_{L}+V P_{R}\right) \psi^{L_{t}} .
\end{aligned}
$$

In this form, the action resembles that of an $L_{t}$-flavour mirror fermion model in the fashion of ref. [58], with $\psi$ the fermion and $\chi$ the mirror fermion field. In fact, for $L_{s}=2$ the hopping terms in $t$ are absent, $\bar{\mu}^{t}=0$ and the model reduces to the mirror fermion model of ref. [58] with equal Yukawa couplings for the fermion and the mirror fermion, and a vanishing single-site mass term. For $L_{s}>2$ the model has a more complicated mass matrix (i.e. non-diagonal couplings among the flavours $s$ or $t$ ) and if the model is going to be more successful in decoupling the mirror fermion than the traditional mirror fermion approach, it must come from this mass term.

The mass matrix for the $L_{t}$ flavours in the model is not diagonal but this can be remedied by more rewriting. First we expand the fermion fields in a plane wave basis, which diagonalizes the Dirac operator and Wilson term, $\psi_{x}^{s}=\sum_{p} e^{i x p} \psi_{p}^{s}, \bar{\psi}_{x}^{s}=\sum_{p} e^{-i x p} \bar{\psi}_{p}^{s}$. Here $\sum_{p}$ is a normalized sum over the momenta on the $d$ dimensional lattice, $\sum_{p} 1=1$. Then we can write,

$$
\begin{aligned}
S_{\psi \chi} & =\sum_{t=1}^{L_{t}} \sum_{p}\left(i \bar{\psi}_{p}^{t} \phi_{p} \psi_{p}^{t}+i \bar{\chi}_{p}^{t} \phi_{p} \chi_{p}^{t}+\bar{\chi}_{p}^{t}\left(w_{p}+\bar{\mu}^{t}\right) \psi_{p}^{t}+\bar{\psi}_{p}^{t}\left(w_{p}+\bar{\mu}^{t}\right) \chi_{p}^{t}\right) \\
& -\sum_{t=1}^{L_{t}-1}\left(\bar{\psi}_{p}^{t} \chi_{p}^{t+1}+\bar{\chi}_{p}^{t+1} \psi_{p}^{t}\right)+\sum_{t}\left(\bar{\psi}_{p}^{t} \chi_{p}^{t}+\bar{\chi}_{p}^{t} \psi_{p}^{t}\right) \\
& -y \sum_{p q}\left(\bar{\chi}_{p}^{t}\left(V_{p-q} P_{L}+V_{q-p}^{\dagger} P_{R}\right) \chi_{q}^{1}+\bar{\psi}_{p}^{L_{t}}\left(V_{q-p}^{\dagger} P_{L}+V_{p-q} P_{R}\right) \psi_{q}^{L_{t}}\right),
\end{aligned}
$$


with $\phi_{p}=\sum_{\mu} \gamma_{\mu} \sin \left(p_{\mu}\right), w_{p}$ the diagonal form of the Wilson term, $w_{p}=\sum_{\mu}\left(1-\cos \left(p_{\mu}\right)\right)$ and $V_{p}$ the Fourier transform of $V_{x}$. For $y=0$ the action has the schematic form

$$
S_{\psi \chi}=(\bar{\psi} \bar{\chi})\left(\begin{array}{cc}
i \phi & M^{\dagger} \\
M & i \phi
\end{array}\right)\left(\begin{array}{l}
\psi \\
\chi
\end{array}\right),
$$

with $M$ a ( $p$ dependent) matrix in flavour space, which can be read off from eq. (91). This action can be diagonalized by making unitary transformations on $\psi$ and $\chi$,

$$
\begin{aligned}
& \omega^{f}=F_{f t}^{\dagger} \psi^{t}, \quad \bar{\omega}^{f}=\bar{\psi}^{t} F_{t f}, \\
& \xi^{f}=G_{f t}^{\dagger} \chi^{t}, \quad \bar{\xi}^{f}=\bar{\chi}^{t} G_{t f},
\end{aligned}
$$

such that $G_{f s}^{\dagger} M_{s t} F_{t g}=\mu_{f} \delta_{f g}$. The matrices $F$ and $G$ are eigenfunctions of $M^{\dagger} M$ and $M M^{\dagger}$ respectively, labeled by the index $f$ :

$$
\left(M^{\dagger} M\right)_{s t} F_{t f}=\left|\mu_{f}\right|^{2} F_{s f}, \quad\left(M M^{\dagger}\right)_{s t} G_{t f}=\left|\mu_{f}\right|^{2} G_{s f}
$$

For suitable choices of the phases of the eigenfunctions, the $\mu_{f}$ 's are real. Substituting the mode expansion (93) into the action (91) with the momentum label restored, we arrive at

$$
\begin{aligned}
& S_{\psi \chi}=\sum_{f=1}^{L_{t}} \sum_{p}\left(\bar{\omega}_{p}^{f} i \phi_{p} \omega_{p}^{f}+\bar{\xi}_{p}^{f} i \phi_{p} \xi_{p}^{f}+\bar{\xi}_{p}^{f} \mu_{p}^{f} \omega_{p}^{f}+\bar{\omega}_{p}^{f} \mu_{p}^{f} \xi_{p}^{f}\right) \\
& -y \sum_{f g, p q}\left[\bar{\xi}_{p}^{f} G_{f 1}^{p \dagger}\left(V_{p-q} P_{L}+V_{q-p}^{\dagger} P_{R}\right) G_{1 g}^{q} \xi_{q}^{g}+\bar{\omega}_{p}^{f} F_{f L_{t}}^{p \dagger}\left(V_{q-p}^{\dagger} P_{L}+V_{p-q} P_{R}\right) F_{L_{t} g}^{q} \omega_{q}^{g}\right] .
\end{aligned}
$$

In this representation of the model, it is seen that all fermion modes $\omega^{f}$ and $\xi^{f}$ interact with the scalar field, but that their effective Yukawa-coupling is determined by the magnitude of their wave function at the waveguide boundaries $t=1$ and $t=L_{t}$. This leads then naturally to the introduction of the two Yukawa-couplings we used in fig.9 to parametrize the phase diagram. There we use the value of the wavefunction of the mirrormode at the waveguide boundary which gives just $y$ and the value of the domain wall fermion at the boundary which is $y e^{-m_{0} L_{s} / 4}$. For $y=0$ the model is seen to describe free, degenerate fermions and mirror fermions with momentum dependent mass $\mu_{p}^{f}$ (for $\mu_{p}^{f} \neq 0$, the eigenstates are $\omega_{p}^{f}+\xi_{p}^{f}$ and $\omega_{p}^{f}-\xi_{p}^{f}$ ). Exactly one flavour, which we denote with $f=0$, has $\mu_{p}^{0}=0$ (up to terms exponentially suppressed in $L_{s}$ ) for $|\hat{p}|<p_{c}$, where $p_{c}$ is the critical momentum defined in sect. 2.1. For $r=1$ and $m_{0}$ close to 1 , the critical momentum is $p_{c} \approx \sqrt{2}$. All other $\mu_{p}^{f}$ and also $\mu_{p}^{0}$ for $p$ outside the critical momentum region, are $O(1)$ in lattice units.

This shows that for $y=0$ and momenta $|\hat{p}| \lesssim p_{c}$, the model contains a massless fermion, $\omega^{0}$, as well as a massless mirror fermion, $\xi^{0}$. All other modes $(f \neq 0)$ as well as the species doublers have a mass of the order of the cutoff. The species doublers of the zeromode $f=0$ are massive because $\mu_{p}^{0}$ is $O(1)$ for momenta with $p_{\mu}= \pm \pi$.

As was discussed already in section 5.2, fig.8 shows the $t$-dependence of the zeromodes $F^{t 0}$ and $G^{t 0}$ of the fermion (indicated by $\psi$ in the figure) and mirror fermion (indicated by $\chi$ ) for 
the smallest momenta $|p|=\pi / L \ll p_{c}$. It shows that the zeromode for the fermion is sharply peaked at $t=\left(L_{t}+1\right) / 2$, i.e. at the domain wall and the zeromode for the mirror fermion is localized at the boundary, at $t=L_{t}$. The non-zeromodes, which are not shown in this figure, are not localized.

\subsection{Large $y$ expansion}

The previous sections introduced the action of the waveguide model and through a mode expansion exhibited its mode spectrum. In addition to the chiral zeromode on the domain wall, new mirror fermion modes appeared at the wave guide boundary. It can be expected that the mirror zeromodes found for $y=0$, which are depicted in fig.8a, remain light and proportional to the vacuum expectation value $v$ for small but non-zero $y$. The interesting question is whether for large values of the Yukawa-coupling the zeromodes at the waveguide boundary become massive and eventually decouple.

As fig. 8 and the discussion of the previous section suggests, things may be simplified by regarding a system with one waveguide only and to consider the effective scalar-fermion model at the waveguide boundary alone. To get some insight into the spectrum at large $y$, one can try to perform a $1 / y$ expansion of the so truncated model [60]. Again the gauge fields will be set to $U=1$. The $1 / y$ expansion is obtained by first relabeling the fermion fields in $s$, while suppressing the even dimensional index

$$
\begin{aligned}
\chi_{s} & =\Psi_{s}, \quad s<s_{0}^{\prime}, \\
\chi_{R s_{0}^{\prime}} & =\Psi_{R s_{0}^{\prime}} ; \quad \chi_{L s_{0}^{\prime}}=\Psi_{L s_{0}^{\prime}+1}, \\
\chi_{s} & =\Psi_{s+1}, \quad s>s_{0}^{\prime} \\
\psi_{R} & =\Psi_{R, s_{0}^{\prime}+1} ; \quad \psi_{L}=\Psi_{L, s_{0}^{\prime}} .
\end{aligned}
$$

By rescaling the field $\psi \rightarrow \frac{1}{\sqrt{y}} \psi$ the action becomes

$$
\begin{aligned}
S_{F} & =\sum_{s}\left(\bar{\chi}_{s} \not \partial \chi_{s}+\bar{\chi}_{s} a(s)(-\Delta+m-1) \chi_{s}\right) \\
& +\sum_{s}\left(\bar{\chi}_{L s} \chi_{R s+1}+\bar{\chi}_{R s+1} \chi_{L s}\right)+\bar{\psi}_{L} V \psi_{R}+\bar{\psi}_{R} V^{\dagger} \psi_{L} \\
& +\sqrt{\alpha}\left[\bar{\psi}(-\Delta+m-1) \chi_{s_{0}^{\prime}}+\bar{\chi}_{s_{0}^{\prime}}(-\Delta+m-1) \psi\right]+\alpha \bar{\psi} \not \partial \psi,
\end{aligned}
$$

where $\psi_{R, L}=P_{R, L} \psi, \chi_{R, L}=P_{R, L} \chi$

$$
\alpha=\frac{1}{y} \text { and } a(s)=1-\delta_{s, s_{0}^{\prime}} .
$$

The sums over $s$ are to be taken in a region around $s_{0}^{\prime}$ neglecting all other defects in the model which are thought of to be far away. In particular the domain wall mass is constant.

In this form it is seen that for $y \rightarrow \infty$, the $\psi$ and the $\chi$ fields decouple. For the $\chi$-fields at the waveguide boundary one may derive the Dirac equation and look for solutions that simultaneously satisfy the massless Dirac equation of the lower dimensional system. Imposing plane 
waves in the even directions the analysis proceeds very similar to the discussion in section 2.1. The Dirac equation reads in momentum space

$$
i \$(p) \chi_{s}+a(s)(m-1-F(p)) \chi_{s}+P_{R} \chi_{s+1}+P_{L} \chi_{s-1}=0 .
$$

Solving eq.(99) for the $\chi$-fields, one arrives at normalizability conditions that dictate the chiral zeromode spectrum on the waveguide boundary [60]. It turns out that in $4+1$ dimensions one finds 7 righthanded and 8 lefthanded zeromodes [60] inside the waveguide, which are bound to the waveguide surface. If the gauge fields are switched on, this leads to a vectorlike spectrum if one takes the zeromode at the domain wall into account.

An important question for a further exploration of the phase diagram is whether the above result is stable against perturbations in $\alpha$. To answer this question we will now compute the $\chi$-field propagator at the waveguide boundary to order $\alpha$. One may start by integrating out the $\psi$-fields in (97) which leads to an effective action

$$
\begin{aligned}
S_{\chi} & =\sum_{s}\left(\bar{\chi}_{s} \not \partial \chi_{s}+\bar{\chi}_{s} a(s)(-\Delta+m-1) \chi_{s}\right)+\sum_{s}\left(\bar{\chi}_{s} P_{R} \chi_{s+1}+\bar{\chi}_{s+1} P_{L} \chi_{s}\right) \\
& -\alpha \sum \bar{\chi}_{s_{0}^{\prime}}(-\Delta+m-1) \sum_{n=0}^{\infty}(-\alpha)^{n}\left[\left(V^{\dagger} P_{R}+V P_{L}\right) \not \partial\right]^{n}\left(V^{\dagger} P_{R}+V P_{L}\right)(\Delta+m-1) \chi_{s_{0}^{\prime}} \\
& =\sum_{s, s^{\prime}} \bar{\chi}_{s} S_{s, s^{\prime}}^{(0)-1} \chi_{s^{\prime}}-\alpha \sum_{x}\left[\bar{\chi}_{s_{0}^{\prime}}(-\Delta+m-1)\right]_{x}\left(V_{x}^{\dagger} P_{R}+V_{x} P_{L}\right)\left[(\Delta+m-1) \chi_{s_{0}^{\prime}}\right]_{x} \\
& +O\left(\alpha^{2}\right) .
\end{aligned}
$$

Here $S^{(0)}$ is the free $\chi$-field propagator which can be computed exactly, see e.g. section 8 . To order $\alpha$, replacing $V$ by its expectation value $v \mathbf{1}$, the Dirac equation reads now

$$
i \not(p) \chi_{s}+a(s)(m-1-F(p)) \chi_{s}+P_{R} \chi_{s+1}+P_{L} \chi_{s-1}-\alpha v(m-1-F(p))^{2} \delta_{s, s_{0}^{\prime}} \chi_{s_{0}^{\prime}}=0
$$

For the symmetric phase with $v=0$ this is exactly the same equation as we found for $y=\infty$. Therefore the result from the large- $y$ expansion is, that in the symmetric phase we will find the same rich zeromode spectrum with equal numbers of left and right handed fermions as we had at $y=\infty$. This is quite in contrast to the anticipated phase diagram, fig.9, where we expected actually a symmetric phase with strong coupling behaviour.

For $v>0$ which corresponds to the broken phase we have an extra piece in the Dirac equation at $s_{0}^{\prime}, \alpha v(1-m+F(p))^{2}$. This gives a mass to the mirror modes of size $v / y$. We see that the weak coupling relation $m_{f} \propto y v$ is replaced by $m_{f} \propto v / y$, i.e. $y \rightarrow 1 / y$. In particular, the fermion masses are still proportional to $v$ and remain light when approaching the phase transition, $v \rightarrow 0$. Passing the phase transition we reach a symmetric phase with a vectorlike zeromode spectrum. In the discussion of the large- $y$ expansions we experienced a first surprise. Since in the large- $y$ expansion all defects are thought to be very far apart from each other, we effectively work in the limit $L_{s} \gg 1$. This means that we are on the $y e^{-m_{0} L_{s} / 4} \approx 0$-axis in the 
phase diagram of fig.9. There we hoped to have the $\mathrm{PMS}_{1}$ phase at $y \gg 1$. Instead we find a second symmetric phase with weak coupling behaviour and an even richer -but vectorlikezeromode spectrum than the one at weak Yukawa-couplings.

\subsection{Reduced model}

The mode expansion discussed in section 5.4 revealed more clearly than the original action (84) what the important modes of the waveguide model are. There is, of course, the domain wall fermion living on the gauged wall. Furthermore, there are mirror fermion like states that live on the waveguide boundary. These states can easily get light and remain in the spectrum. We saw that this can happen for weak coupling as well for very large coupling, which led to a revision of the phase diagram in fig.9. However, at intermediate values of $y$, there might still be still our candidate phase, $\mathrm{PMS}_{1}$ where we can hope to construct a chiral gauge theory on the domain wall at the end. It became clear that it is very natural to think of the model as a mirror fermion model along the lines of [58] and that it would be the peculiar mass matrix which would be responsible for decoupling the mirror fermions in contrast to the normal approach [58].

As we will see below, numerical simulations in the waveguide model even in the quenched approximation become very demanding. On the other hand we could identify the important building blocks of the domain wall model, the behaviour of which make the model succeed or let it fail, uniquely. These building blocks are the domain wall mode and the waveguide mode inside the waveguide. Furthermore, these modes appear only in the low energy regime below the critical momentum $p_{c}$. Above $p_{c}$ they become heavy and delocalize.

One may therefore attempt to construct a reduced model that shows the characteristic properties of the waveguide model with these ingredients. As we saw in section 5.5, the anticipated fermion spectrum for the reduced model is not realized at very strong values of $y$. Therefore the reduced model only makes sense under the assumption that at intermediate $y$-values the $\mathrm{PMS}_{1}$ phase really exist. We will use for this "reduced" model the domain wall zeromode $\omega^{0}$, the mirror mode $\xi^{0}$ at the waveguide boundary, the scalar fields $V$ and the critical momentum $p_{c}$. This leads to a model

$$
S^{\text {red }}=\sum_{|\hat{p}|<p_{c}}\left[i \bar{\omega}_{p}^{0} \phi_{p} \omega_{p}^{0}+i \bar{\xi}_{p}^{0} \phi_{p} \xi_{p}^{0}\right]+y \sum_{|\hat{p}|,|\hat{q}|<p_{c}} \bar{\xi}_{p}^{0}\left(V_{q-p}^{\dagger} P_{R}+V_{p-q} P_{L}\right) \xi_{q}^{0}
$$

In this reduced model the domain wall fermion $\omega^{0}$ is completely decoupled from the mirror fermion $\xi^{0}$ and the scalar field $V$. The mirror mode is then coupled to the scalar field with a strength of the Yukawa-coupling $y$. We now have exactly the situation which might lead to a successful description of a chiral theory if the waveguide model would work: An unaffected chiral zeromode on the domain wall and a Yukawa-like theory on the waveguide boundary. The only question is, whether this Yukawa-like theory possesses the strong symmetric phase or not. One may argue that the above model is an oversimplification of the full theory. But one can give plausible arguments that this kind of reduction makes sense 25]. We will see further from 
the numerical simulations that the reduced model behaves almost the same as the full model. This is so at least at the values of $y$ where the simulations discussed in the next section have been performed. We know, of course, from the large- $y$ expansion, that the reduced model does not represent the full model at $y \gg 1$. Third, if in the reduced model no $\mathrm{PMS}_{1}$ phase exists, it will also not exist in the full model. The only addition from the full model would be that the domain wall zeromode and the mirror mode start to interact. But then they either combine to form a massive Dirac particle or the effective interaction is so weak that we are back in the situation of the reduced model. The additional heavy modes in the model, in particular the modes above the critical momentum $p_{c}$, do not play an important role as their coupling can be expected to be inversely proportional to their mass and hence is weak.

\section{$6 \quad$ Numerical simulations in the waveguide model}

Up to now we discussed the waveguide model at the two boundaries of the phase diagram for small and large Yukawa-couplings. In both these limits it seems not to be possible to construct a chiral gauge theory. This may, however, still be possible in the middle of the phase diagram at intermediate values of the Yukawa-coupling. In this region both, the weak coupling as well as the strong coupling expansion do not work. Therefore, in order to answer the question whether the $\mathrm{PMS}_{1}$ phase exists one has to rely on numerical simulations.

As already mentioned earlier, Yukawa models in 2 and 4 dimensions have been subject to extensive investigations in the last few years. In these studies it became clear that they could either show a $\mathrm{PMS}_{1}$ phase [59] or not [61], depending on the choice of the Yukawa-coupling. Two examples of these kind of Yukawa models are the local coupling

$$
S_{l c}=y \sum_{x} \bar{\psi}_{x}\left(V_{x} P_{R}+V_{x}^{*} P_{L}\right) \psi_{x}
$$

and the hypercubic coupling

$$
S_{h c}=y \sum_{x} \frac{1}{4} \sum_{b} \bar{\psi}_{x}\left(V_{x-b} P_{R}+V_{x-b}^{*} P_{L}\right) \psi_{x}
$$

where we only show the Yukawa-part of the action. In the case of the hypercubical coupling the sum over $b$ means a sum over all points of a lattice hypercube surrounding the point $x$. The model with the local coupling is known to have a PMS phase whereas the one with hypercubical coupling does not.

A form of the Yukawa-coupling with a momentum cut-off as in the waveguide model has so far not been investigated. Therefore the question whether a $\mathrm{PMS}_{1}$ phase exists or not remains undecided. Unfortunately the special form of the Yukawa-coupling as given in the wave guide model and in its reduced form prohibit an analytical treatment for intermediate values of $y$. Obviously a numerical study of the 5-dimensional system would be very demanding. It turns out, however, that the strong coupling behaviour we are after already appears in 2-dimensional 
Yukawa models. Even more, the phase diagrams of 2 and 4-dimensional models are very similar so that it is a reasonable strategy to search for the $\mathrm{PMS}_{1}$ phase in the waveguide model in 2 dimensions.

This path has been followed in 25]. Most results are obtained in the quenched approximation, neglecting all fermion loops. This seems to be sufficient for the question whether a PMS phase exists. Although in the quenched approximation the phase transition lines shown in fig.9 do not really exist, the model should show typical weak or strong coupling behaviour. The experience from earlier studies of Yukawa models suggests two approaches to identify or exclude a strong coupling symmetric phase. The first is the fermion mass spectrum and the second the eigenvalue spectrum. Both show characteristic behaviour if a strong coupling phase exists. The results of this numerical investigation will be discussed in the next two subsections.

The $(2+1)$-dimensional model is simulated using $\mathrm{U}(1)$ scalar fields. The pure bosonic sector of the model consists therefore of a $X Y$-model on the boundary of the waveguide region. One might worry that the $X Y$-model we are going to look at is too different from the real 4-dimensional system we are interested in. There is a phase transition from a spin-wave to a Kosterlitz-Thouless phase at $\kappa \approx 0.5$ which is certainly in a different universality class compared to the phase transition in four dimensions. In addition there is no spontaneous symmetry breaking in the $X Y$-model. However, if on the finite lattice a vacuum expectation value is defined through the standard rotation technique [62,

$$
v=<\frac{1}{L^{2}}\left|\sum_{x} V_{x}\right|>
$$

then the so defined vacuum expectation value $v$ is zero in the Kosterlitz-Thouless phase but $v>0$ in the spinwave phase. In this way the 2-dimensional $X Y$-model fakes the situation that appears in spontaneous symmetry breaking in 4 dimensions. Increasing the lattice volume, $v$ approaches zero also in the spinwave phase as it should. For a finite lattice we expect therefore that the fermion mass follows the perturbative relation $m_{F} \propto y v$, if the volume is not too large. We will in the following refer to the spinwave phase as the broken and to the Kosterlitz-Thouless (or Vortex) phase as the symmetric phase.

All simulations presented below have been performed in a $(2+1)$-dimensional setup with a lattice of size $L^{2} L_{s}$ with $L=12, L_{s}=26$ being typical values of the lattice extensions. The Wilson parameter $r$ has always been set to $r=1$ and the domain wall height was chosen to be

$m_{0}=1$, too. Typically several thousand scalar field configurations have been obtained using a cluster algorithm. The fermionic quantities have been obtained by standard techniques like Conjugate Gradient or Lanczos. In order to avoid convergence problems of these algorithms antiperiodic boundary conditions for one of the 2-dimensional indices have been chosen.

\subsection{Mass spectrum}

The fermion spectrum is a first indication for the presence of strong coupling behaviour. Fermion masses can be obtained from the propagator 


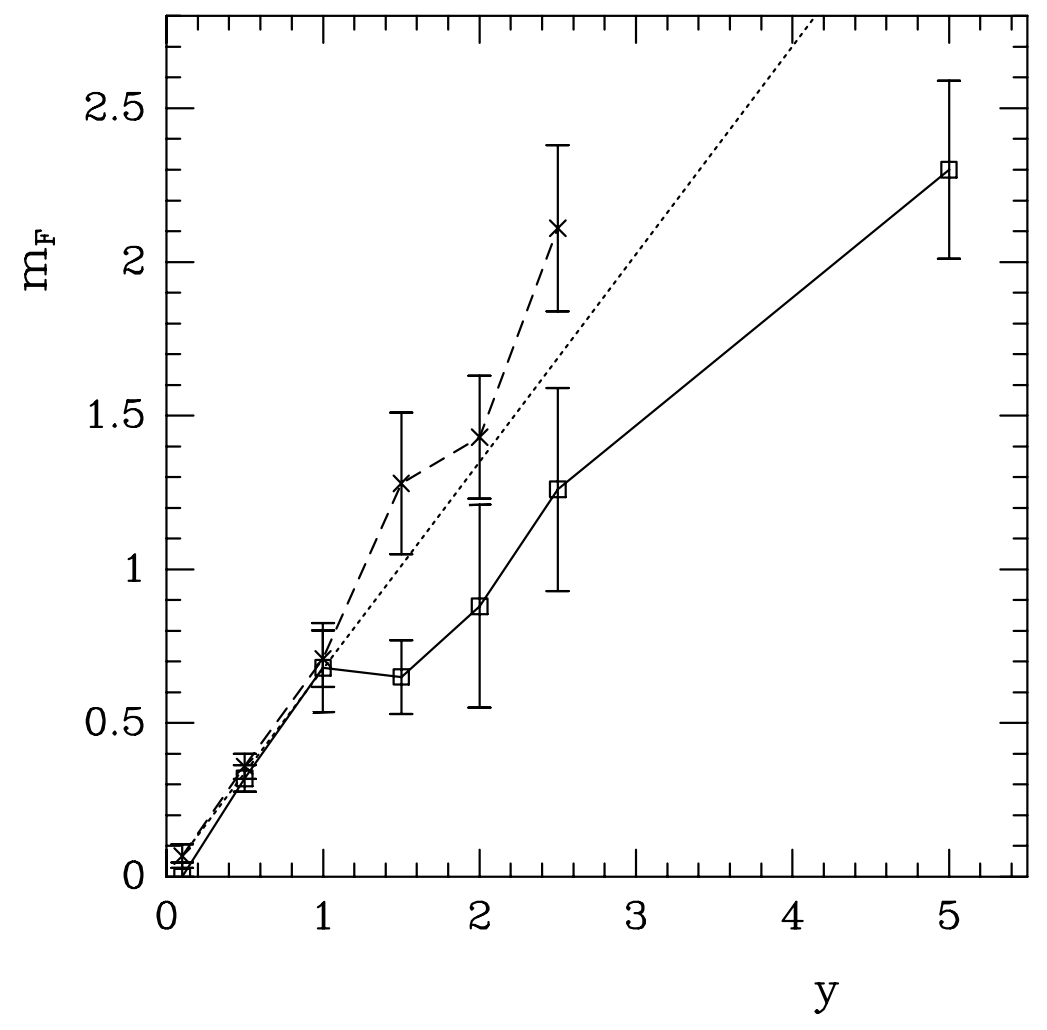

Figure 10: The fermion mass of the fermion at the waveguide boundary as a function of the Yukawa-coupling y. Both, the fermion in the full model (boxes) and the reduced model (crosses) are consistent with a linear behaviour, in particular at small $y$. The dotted line is $y v$. All results are at $\kappa=0.5$.

$$
S^{s t}(p)=L^{-2} \sum_{x y} e^{i p(x-y)}<\Psi_{x}^{s} \bar{\Psi}_{y}^{t}>,
$$

which can be computed using the conjugate gradient algorithm to find matrix elements of the inverse fermion matrix on a scalar field background. Using the symmetry

$$
S_{R R}^{s t}(p)=S_{L L}^{L_{t}+2-s, L_{t}+2-t}(P p),
$$

statistics can be enlarged by measuring the $L L$ and the $R R$ components and averaging over them. Fermion masses are finally obtained by a fit to a free fermion propagator ansatz

$$
S(p)_{R R(L L)}=-i Z_{F}\left(\sin \left(p_{1}\right)-(+) i \sin \left(p_{2}\right)\right) /\left(\sum_{\mu} \sin ^{2}\left(p_{\mu}\right)+m_{F}^{2}\right) .
$$

The numerical results can be perfectly described by the ansatz (108) (see fig.5 in [25]).

What do we expect for the fermion masses having the phase diagram of fig.9 in mind? Weak coupling behaviour For small values of the Yukawa-coupling $m_{f} \propto y v$ with $v$ the vacuum expectation value of the scalar field using (105). The proportionality of the fermion mass to $y$ could be confirmed in [25], see fig.10. In the symmetric phase of the model the fermion mass turns out to be zero. This excludes the small $y$ region for a construction of a chiral gauge theory as the mirror modes can not be decoupled. 


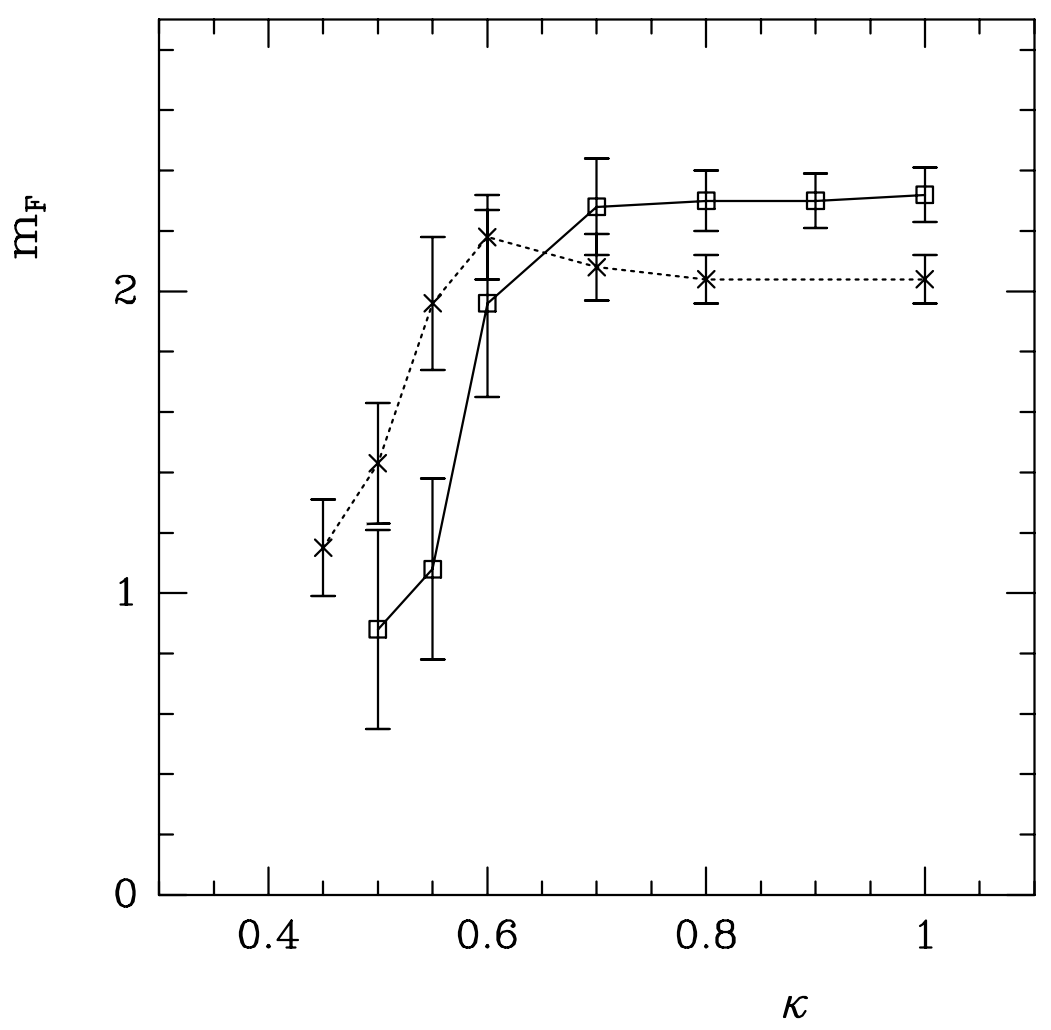

Figure 11: The fermion masses from numerical simulations at $y=2$ as function of $\kappa$. The boxes correspond to the full and the crosses to the reduced model.

strong coupling behaviour For larger values of the Yukawa-coupling such that one does not enter the region where the large- $y$ expansion of section 5.5 is valid we hope to find our favourite $\mathrm{PMS}_{1}$ phase. From experience with models showing the strong coupling phase, we expect the fermion mass to follow the mean field strong coupling behaviour

$$
m_{F} \propto z^{-2} ; z^{2}=\operatorname{Re}\left\langle V_{x} V_{x+\mu}^{*}\right\rangle
$$

The quantity $z$ is non-zero in the symmetric as well as in the broken phase. It is monotonically decreasing with decreasing $\kappa$. In the $\mathrm{PMS}_{1}$ phase the fermion mass measured from the numerical simulation would follow the behaviour in (109) and would therefore increase with decreasing $\kappa$. In fig.11 the behaviour of the fermion mass as a function of $\kappa$ is shown at $y=2$ where one would expect the strong coupling behaviour to show up if the model would behave similar to 2-dimensional Yukawa models with local coupling.

In the figure, the boxes, connected by a dotted line to guide the eye, correspond to the full waveguide model, the crosses to the reduced model. The masses from both models are very similar. They stay first almost constant at high values of $\kappa$ and then start to decrease when $\kappa$ is lowered. This behaviour is in sharp contrast to the expected increase of the masses if there would be a strong coupling region. Unfortunately, the numerical simulations could not be driven into the symmetric phase $\left(\kappa<\kappa_{c}=0.5\right)$. The propagators became too noisy and no reliable mass value could be obtained. 
Nevertheless the picture is in clear contradiction with the scenario we have hoped for. The mirror fermion masses become small approaching the critical line. This is another indication that a $\mathrm{PMS}_{1}$ phase does not exist in the waveguide model. Quite in contrary, the fact that fermions seem to become light in the symmetric phase at $y=2$ point into the direction that the fermion spectrum shows weak coupling behaviour. One other important aspect of fig.10 and fig.11 is, that they show the resemblance of the reduced model to the full model. This similarity will be relied on when in the next subsection the eigenvalue spectrum is determined, which will give an additional hint that the $\mathrm{PMS}_{1}$ phase in the waveguide model is missing.

\subsection{Eigenvalue spectrum}

A complementary way to detect a strong coupling phase is the qualitative behaviour of the eigenvalue spectrum for different Yukawa models [63]. The shape for a Yukawa-model with local (103) and hypercubical (104) couplings are quite characteristic. It would, of course be ideal to compute the eigenvalues in the full domain wall model. However, the matrix from which the eigenvalues have to be determined is of size $2 L_{s} L^{2} \otimes 2 L_{s} L^{2}$. To get all eigenvalues of this matrix is almost impossible for reasonably large lattice sizes. We can, however, rely on the similarity of the reduced model to the full waveguide model and try to determine the eigenvalues in the reduced model at different values of $y$.

In fig.12 the eigenvalue spectra for the reduced domain wall model (a), the model with local (b) and with hypercubical (c) Yukawa-coupling is shown. They have been obtained at $y=0.2,1$ and 4 from left to right and were averaged over 5 scalar field configurations. The lattice size has been $L=12$. For the waveguide model the eigenvalues at small Yukawa-coupling are very similar for all three models and form a narrow band. For $y=1$ there appear some differences in that for the waveguide and the hypercubical model the band broadens whereas in the model with local Yukawa-coupling they seem to develop a gap.

The difference becomes much more pronounced for $y=4$. The local Yukawa-coupling model shows a ring structure typical for a strong phase [63]. On the other hand it is all too obvious that the domain wall model follows the trend of the hypercubical model. Even at $y=4$ which is a quite strong Yukawa-coupling, no sign of strong coupling behaviour can be seen. The eigenvalue distribution just broadens with increasing $y$. The good representation of the full

model by the reduced one suggested in figs.10 and 11 leads to the conclusion that also in the full domain wall model the eigenvalues behave very similar.

This is strengthened by the behaviour of the number of conjugate gradient iterations to reach a given residue. This is determined by the condition number of the fermion matrix. In the model with a local coupling which has the strong symmetric phase, the number of conjugate gradient inversions first rises with increasing $y$ but then, when entering the strong coupling region it decreases again, due to the fact that the fermions become massive. No such decrease can be seen in the full waveguide model. The number of conjugate gradient inversions just 
with $v>0$. This pictures is motivated by the ordering effect of the fermions which shift the phase transition of the pure bosonic theory towards smaller values of $\kappa$. Increasing $y$ even further, $v$ becomes zero for some critical Yukawa-coupling $y_{c}$, if one enters a second symmetric phase. Whether this is a phase with strong coupling behaviour could then be clearified by computing the fermion spectrum.

This scenario can be tested by numerically computing $v$ using the rotation technique. Unfortunately, for medium size values of $y \approx 1$, the step size in the Hybrid Monte Carlo algorithm had to be decreased by an order of magnitude resulting in large autocorrelation times. This became even worse when increasing $y$ further such that no reliable simulation could be performed in the interesting $y$ region. The bad behaviour of the Hybrid Monte Carlo algorithm is, however, consistent with the development of the eigenvalue spectrum discussed in the previous section. If a strong symmetric phase exists one would expect the behaviour of the Hybrid Monte Carlo algorithm to become much better again since the fermion would have become massive. It might be an interesting prospect to attack the difficult region in $y$ again by using the recently suggested new simulation algorithm for fermions by Lüscher 65.

\section{Infinite extra dimension}

As we have seen in the previous sections, our attempts to achieve a chiral gauge theory with domain wall fermions seem to fail. The effect of the anti-domain wall can not be switched off in case of 5-dimensional gauge fields. In the waveguide model where gauge fields are kept 4dimensional the anti-wall can be shielded from the wall but only for the expense of introducing a wave guide wall. New light mirror fermion modes appear on this wall rendering the theory vectorlike again.

We found on the other hand in section 2.1 that on the infinite lattice there is only one chiral zeromode and no necessity for introducing a second wall emerges. One might first discard an infinite lattice as not leading to a practical proposal for simulations. This is certainly the case for the euclidean action formulation of a theory. Narayanan and Neuberger [29, 66] suggested to take a different route of thinking. They regard the extra dimension as the "time-axis" of a $(4+1)$-dimensional system and propose to study the 4-dimensional Hamiltonian of this theory. The extra dimension is thought of being kept strictly infinite and as such it fulfills the sole purpose to project onto the ground state of the system. There will be two Hamiltonians $H^{ \pm}$ corresponding to the two sides of the wall and accordingly there will also be two ground states $\mid 0 \pm>$. The theory can then be defined as the overlap of these two ground states.

Also in this approach the gauge fields are kept 4-dimensional. As opposed to the waveguide model there is no need of adding an extra wall and thus no additional fields have to be introduced. To keep the extra dimension infinite may also be motivated from the index of the fermion matrix $M$. What one would like to achieve in order to obtain a chiral theory is that the fermion matrices $M$ and $M^{\dagger}$, belonging to the left and righthanded modes, respectively, 
have different indices. In particular, $M$ should have index 1 and $M^{\dagger}$ index 0 , which would correspond to a single chiral fermion as an eigenstate of $M$. Such a situation can only be reached when the matrices $M$ and $M^{\dagger}$ are infinite. Of course, in the domain wall model with a finite $M$ it was hoped that one can keep both chiral modes on the lattice such that they can be separated and shielded from each other. The model proposed in [29] can be considered as a combination of domain wall fermions as suggested by Kaplan [13 and the idea of infinitely many regulator fields advocated by Frolov and Slavnov 67. There it was suggested to introduce infinitely many Pauli-Villars fermion fields to regulate a chiral gauge theory. Interpreting the extra dimension as a flavour space, the different flavours can be associated with new fields that have been introduced. Choosing the extra dimension to be infinite, these new fields play the role of the infinitely many Pauli-Villars fields in the approach of [67].

To be specific, we will consider the following 4-dimensional action

$$
\begin{aligned}
S_{F} & =\sum_{x, s} \sum_{\mu=1}^{4} \bar{\psi}_{x}^{s}\left[\left(\not \partial(U)_{x}-\Delta_{x}(U)+m(s)\right] \psi_{x}^{s}\right. \\
& +\frac{1}{2} \sum_{x, s} \bar{\psi}_{x}^{s}\left[\delta_{s, s+\mu_{5}}\left(1+\gamma_{5}\right)+\delta_{s-\mu_{5}, s}\left(1-\gamma_{5}\right)-2 \delta s, s\right] \psi_{x}^{s} .
\end{aligned}
$$

This action corresponds to the action (78) with the 5-dimensional gauge fields $V$ set to unity and the Wilson coupling $r$ set to $r=1$. The domain wall mass is chosen as

$$
m(s)=d-m \operatorname{sign}\left(s+\frac{1}{2}\right)
$$

with $d$ the (odd) dimension of the system. Note that the wall in this setup has been chosen to be in the middle of the link connecting the regions in $s$ with positive and negative domain wall mass. Of course, keeping the summation over $s$ infinite leads to divergences. However, these divergences come from the bulk and one may define an interface effective action

$$
S_{i}(U)=S_{e f f}(U)-\frac{1}{2}\left[S_{e f f}^{+}(U)+S_{e f f}^{-}(U)\right] .
$$

$S_{\text {eff }}^{ \pm}(U)$ are the bulk actions for a constant domain wall mass to the right and the left of the location of the domain wall. The interface effective action is defined on the wall. It is now finite and can be implemented order by order in perturbation theory.

Staying in the euclidean action formulation would, however, not lead to any practical way for numerical simulations. The path Narayanan and Neuberger are following is to construct $S_{i}(U)$ from a vacuum overlap formula. This is obtained in a first step by constructing the transfer matrix $\hat{T}=e^{\hat{H}}$ (with the lattice spacing $a=1$ ) from the lattice Hamiltonian $\hat{H}, \hat{H}=\hat{a}^{\dagger} H \hat{a}$ with $\hat{a}^{\dagger}$ and $\hat{a}$ are the usual fermionic creation and annihilation operators and $H$ is represented by a matrix, the explicit form of which will be given below.

For Wilson fermions with $r=1$ the transfer matrix describes the evolution of the system from one timeslice to the next and therefore over the smallest distance on the lattice. As the transfer matrix contains the full physical information of a system, a description of a model in 
terms of the transfer matrix is completely equivalent to its path integral formulation. Indeed, the path integral $Z$ may be written with the usual trace formula $Z=\operatorname{Tr} \hat{T}^{N}$ when $N$ is the extension of the lattice in the "time" direction. The construction of the transfer matrix, which is a non-trivial task, was performed by Narayanan and Neuberger, following [68 closely. They find the transfer matrices on both sides of the wall, $\hat{T}_{+}$and $\hat{T}_{-} \cdot \hat{T}_{+}$and $\hat{T}_{-}$are the main building blocks of the theory in the infinite extra dimension formulation and for the construction of the overlap formula.

As a result a representation of the interface effective action in terms of the transfer matrices can be given

$$
e^{S_{i}(U)}=\lim _{s \rightarrow \infty} \frac{<b-\left|\left(\hat{T}_{-}\right)^{s-1}\left(\hat{T}_{+}\right)^{s-1}\right| b+>}{\sqrt{<b-\left|\left(\hat{T}_{-}\right)^{2 s-2}\right| b-><b+\left|\left(\hat{T}_{+}\right)^{2 s-2}\right| b+>}}
$$

where $<b \pm \mid$ denote some boundary conditions at $s= \pm \infty$. Choosing these boundary conditions as

$$
|b \pm>=| 0 \pm>
$$

in the $s \rightarrow \infty$ limit the ground state is projected out in (113) and the interface effective action is given by a simple overlap formula

$$
e^{S_{i}(U)}=<0-\mid 0+>
$$

To get a feeling for the formula (115) which corresponds to the -infinite- lattice effective action, we will now as a first step discuss its continuum limit for a $(2+1)$-dimensional system. We will check in continuum perturbation theory whether we will find the anomaly we expect, if the overlap formula correctly describes the chiral gauge theory it was constructed for. The Hamiltonians for the left and the right sides of the wall, $H_{-}$and $H_{+}$, respectively, are in momentum space

$$
-H_{ \pm}(p, q)=H_{0}^{ \pm}(p) \delta_{p, q}+\int \frac{d^{2} k}{(2 \pi)^{2}}\left[z_{k} \tau \delta_{q, p+k}+z_{k}^{*} \tau^{\dagger} \delta_{q, p+k}\right]
$$

where the unperturbed Hamiltonians $H_{0}^{ \pm}$are diagonal in momentum space

$$
\begin{gathered}
H_{0}^{ \pm}(p)=\left(\begin{array}{cc}
\mp m & p_{1}-i p_{2} \\
p_{1}+i p_{2} & \pm m
\end{array}\right) \\
\tau=\left(\begin{array}{ll}
0 & 0 \\
1 & 0
\end{array}\right)
\end{gathered}
$$

and

$$
z_{k}=\int d^{2} x e^{i k x}\left[A_{1}(x)+i A_{2}(x)\right] .
$$

If we denote by $\Psi_{p}^{ \pm}$the set of eigenvectors belonging to positive eigenvalues of the full Hamiltonian

$$
-H_{ \pm} \Psi_{p}^{ \pm}=\Lambda_{p}^{ \pm} \Psi_{p}^{ \pm} ; \Lambda_{p}^{ \pm}>0
$$


we can define an overlap matrix as

$$
O_{p q}=\Psi_{p}^{-\dagger} \Psi_{q}^{+} .
$$

The continuum interface effective action is then given by

$$
e^{S_{i}(A)}=\operatorname{det} O
$$

To proceed we will now sketch the perturbative evaluation of $S_{i}(A)$. For this purpose we expand the fields

$$
\begin{aligned}
& \Psi_{p}^{+}=\psi_{p}^{+}+\psi_{q}^{+} A_{q p}+\chi_{q}^{+} B_{q p} \\
& \Psi_{p}^{-}=\psi_{p}^{-}+\psi_{q}^{-} C_{q p}+\chi_{q}^{-} D_{q p}
\end{aligned}
$$

where $\psi^{ \pm}$and $\chi^{ \pm}$are the eigenvectors corresponding to the positive and negative eigenvalues of the unperturbed Hamiltonian $H_{0}^{ \pm}$, respectively. $A, B, C$ and $D$ are coefficients which are linear in the gauge fields. The eigenvalues are also expanded

$$
\Lambda_{p}^{ \pm}=\lambda_{p}+\lambda_{p}^{ \pm(1)}
$$

with $\lambda_{p}$ the eigenvalues belonging to the free Hamiltonian. The perturbation Hamiltonian can be read off from (116)

$$
-H_{1}(p, q)=\left[z_{k} \tau \delta_{q, p+k}+z_{k}^{*} \tau^{\dagger} \delta_{q, p+k}\right]
$$

and the coefficients and the eigenvalues can be obtained from standard Schrödinger perturbation theory

$$
\begin{aligned}
\lambda_{p}^{ \pm(1)} & =\psi_{p}^{ \pm \dagger} H_{1} \psi_{p}^{ \pm} \\
A_{p q} & =\frac{\psi_{p}^{+\dagger} H_{1} \psi_{q}^{+}}{\lambda_{q}-\lambda_{p}} p \neq q \\
B_{p q} & =\frac{\chi_{p}^{+\dagger} H_{1} \psi_{q}^{+}}{\lambda_{q}+\lambda_{p}} \\
C_{p q} & =\frac{\psi_{p}^{-\dagger} H_{1} \psi_{q}^{-}}{\lambda_{q}-\lambda_{p}} p \neq q \\
D_{p q} & =\frac{\chi_{p}^{-\dagger} H_{1} \psi_{q}^{-}}{\lambda_{q}+\lambda_{p}} .
\end{aligned}
$$

The diagonal terms $A_{p p}$ and $C_{p p}$ in (126) are undetermined. This can be partly repaired by imposing orthonormality of the eigenvectors which leads to

$$
A_{p p}+A_{p p}^{*}=0 ; C_{p p}+C_{p p}^{*}=0
$$

which fixes the real part of both coefficients. However, the imaginary part of $A$ and $C$ remains undetermined and therefore there is a phase ambiguity in the interface effective action. This phase ambiguity reflects the fact that it is the imaginary part of the complex action where the 
chiral nature is hidden [69]. The real part of the action is vectorlike. This can also be seen from (113). Taking there periodic boundary conditions in the extra $s$ direction, in the limit $s \rightarrow \infty$

$$
e^{S_{i}(U)}=|<0-| 0+>\left.\right|^{2} .
$$

The effective action (128) is real and corresponds to the domain wall setup with two chiral zeromodes of opposite chirality. Thus we see that the imaginary part of the action is the crucial ingredient for chiral gauge theories. One proposal to compute the imaginary part of the action can be found in [70].

The still undetermined phases for the interface effective action have to be fixed. This can be done in perturbation theory by demanding that the projection of the true eigenstate onto the perturbed one $\Psi_{p}^{ \pm^{\dagger}} \psi_{p}^{ \pm}$being real. This amounts to Brillouin-Wigner perturbation theory. Of course, also for a non-perturbative investigation of a chiral gauge theory the phase ambiguity appears. The authors in [29] propose to take the same phase fixing condition as in perturbation theory. The advantage of this condition is that it can be easily implemented and thus the theory is completely determined. It is, however, an important -and still open- question whether the Brillouin-Wigner condition is suitable also for non-perturbative and strongly fluctuating gauge field configurations.

To arrive eventually at the anomaly one has to go to second order in perturbation theory. This can be done [29] and as a result the anomaly is given as

$$
\sum_{\mu} \partial_{\mu} \frac{\delta I}{\delta A_{\mu}(x)}
$$

where

$$
I=\frac{1}{16 \pi} \sum_{p}\left[\frac{p_{1}+i p_{2}}{p_{1}-i p_{2}} z_{p}^{*} z_{-p}^{*}-\frac{p_{1}-i p_{2}}{p_{1}+i p_{2}} z_{p} z_{-p}\right]
$$

and $z$ is given in (119),

$$
z_{p}=A_{1, p}+i A_{2, p} ; z_{-p}^{*}=A_{1, p}-i A_{2, p} .
$$

Thus the anomaly becomes

$$
\frac{1}{4 \pi}\left(p_{2} A_{1, p}-p_{1} A_{2, p}\right)=-\frac{1}{4 \pi} F_{12}(p) .
$$

Eq. (132) is the continuum form for the anomaly on the wall from the overlap formula (115). Since in deriving this equation the variation of the action with respect to the gauge fields was taken for the effective interface action we should have expected to find the anomaly which corresponds to the chiral mode on the wall. This would be the consistent anomaly as we discussed in section 4 . In fact, the strength of the anomaly in (132) is $1 / 4 \pi$ which is just half of the value of the covariant anomaly we have discussed earlier for the domain wall system. Thus it corresponds to the consistent anomaly which is the correct result. 
As a next step it would be certainly interesting to see, whether the overlap formalism can be taken over to the lattice. The transfermatrix can be explicitly constructed

$$
e^{H_{ \pm}}=\left(\begin{array}{cc}
\frac{1}{B^{ \pm}} & \frac{1}{B^{ \pm}} C \\
C^{\dagger} \frac{1}{B^{ \pm}} & C^{\dagger} \frac{1}{B^{ \pm}} C+B^{ \pm}
\end{array}\right) .
$$

The matrices $B$ and $C$ depend on the gauge fields and their explicit forms are

$$
\begin{gathered}
B^{ \pm}=(d+1 \mp m)-\frac{1}{2} \sum_{\mu}\left[\delta_{x, x+\mu} U_{x, \mu}+\delta_{x, x-\mu} U_{x-\mu, \mu}^{*}\right] \\
C=\frac{1}{2} \sum_{\mu}\left[\delta_{x, x+\mu} U_{x, \mu}-\delta_{x-\mu, \mu} U_{x-\mu, \mu}^{*}\right] \gamma_{\mu} .
\end{gathered}
$$

The effective action on the interface is

$$
e^{S_{i}(U)}=\frac{U<0-\mid 0+>_{U}}{1<0-\mid 0+>_{1}} e^{i\left[\Phi_{+}(U)-\Phi_{-}(U)\right]} .
$$

The $\mid 0 \pm>_{U}$ are the ground states of the Hamiltonian in presence of the gauge fields whereas $\mid 0 \pm>_{1}$ denote the ground states for free fermions. The phases $\Phi_{ \pm}(U)$ are undetermined which again reflects the phase ambiguity we have encountered in (126,127). The phases are fixed using the same condition as in perturbation theory also for the non-perturbative case

$$
e^{i \Phi_{ \pm}(U)}=\frac{U<0 \pm \mid 0 \pm>_{1}}{|U<0 \pm| 0 \pm>_{1} \mid}
$$

In close analogy to the earlier discussion of the anomaly an external gauge field is chosen

$$
U(k)=e^{i \frac{A_{\mu}(\phi)}{L} \cos \left(\frac{2 \pi k}{L}\left(n+\frac{1}{2}\right)\right)}
$$

with

$$
A_{\mu}(\phi)=A_{\mu}+2 \phi \sin \left(\frac{\pi k}{L}\right)
$$

The anomaly equation now reads

$$
-\left.\frac{i}{\pi} \lim _{L \rightarrow \infty} \frac{\partial S\left(A_{\mu}(\phi)\right)}{\partial \phi}\right|_{\phi=0}=-\frac{1}{4 \pi}\left[A_{2} k_{1}\right]
$$

In order to compute the anomaly numerically, the Dirac sea has to be filled and the eigenfunctions corresponding to the negative energy eigenstates have to be found. This amounts to find the eigenvalues of (133) numerically. In the computation $A_{2}$ was taken to be $A_{2}=0.32$. In [29] two values of the domain wall mass were chosen, $m=0.5$ and $m=0.9$ and the anomaly was computed on different size lattices. At both $m$ the value of the anomaly was extrapolated to $L=\infty$. Both extrapolated values coincide and give $-0.02545(5)$. This is to be compared to the value of the anomaly in the continuum of -0.02546 . The same test of the anomaly equation to be fulfilled on the finite lattice can also be performed in four dimensions by a clever choice of the external gauge fields keeping the Hamiltonian to be at least block diagonal. Again the 
continuum value of the strength of the anomaly could be reproduced in the thermodynamic limit.

We discussed in this section a proposal of keeping the extra dimension in the domain wall model strictly infinite. Alternatively we might think of having introduced infinitely many regulator fields for a chiral gauge theory. We saw, that in continuum perturbation theory the correct value of the consistent anomaly is found. Even more remarkable is that the overlap formula resulting from the ground state projection can be implemented on the finite lattice. The task there is to diagonalize a -admittedly huge- matrix. Choosing external gauge fields that vary smoothly, the value of the consistent anomaly could be reproduced when the finite lattice results are extrapolated to the infinite lattice. With suitable techniques of matrix diagonalization a numerical simulation may be reached eventually.

With this outlook it might be possible to test whether a chiral gauge theory can be obtained when the gauge fields are made dynamical. Of course, the tests being performed on the overlap formula so far correspond to the situation in the domain wall model where everything worked. There we also found for smooth external gauge fields that the correct -in this case covariant anomaly - can be reproduced on the lattice and that the chiral zeromode spectrum comes out as desired. This encouraging picture was only destroyed when dynamics had been added. Therefore it seems that only a full simulation can decide the question whether the infinite $s$ approach is the solution of the chiral fermion problem on the lattice.

In a numerical simulation only the real part would be used for the updating. Given a new gauge field configuration consisting of a change of only one link, the imaginary part would be computed by diagonalizing $H_{ \pm}$. It is then hoped that, since only one link is changed at a time, the old eigenfunction of $H_{ \pm}$is a good starting point for the new diagonalization process. The general idea is not to take the imaginary part in the updating process but into the measured observables. It is, of course, not clear at all that the fluctuations of the imaginary part for realistic lattice gauge field configurations are small. If these fluctuations are large, which might well be possible due to the roughness of the 4-dimensional gauge fields, this would not lead to any practical simulation algorithm. On the other hand an implementation of the above simulation proposal could provide the first measure for the strength of the fluctuations of the imaginary part of chiral fermion actions.

One might find the idea unsatisfactory that a chiral theory can only be constructed by considering the Hamiltonians for both sides of the wall and construct the overlap of the corresponding ground states. There seems to be at the moment no way of connecting the overlap setup with any euclidean lattice formulation of the same theory. However, the anomaly in the infinite dimensional lattice approach for chiral lattice fermions can be understood from a limiting procedure of a lattice version with finite extend in the $s$-direction 45. In this version the $s$-direction is split into a gauged region for $s<N$ and an ungauged region for $N<s^{\prime}<N^{\prime}<\infty$. The limiting procedure which gives the correct - consistent- anomaly is to let both $N$ and $N^{\prime}$ go to infinity simultaneously. Therefore the interface effective action (112) 
might be understood as the limit $s \rightarrow \infty$ of a gauge variant finite lattice version. As we have seen in the discussion of the waveguide model, section 5.2, a gauge variant action can be made gauge invariant by adding scalar or Stückelberg fields. But then the infinite $s$ effective action is hiding that it came from the waveguide model on the finite lattice. As the waveguide model failed in constructing a chiral gauge theory, the same faith may await the overlap approach to chiral fermions on the lattice.

\section{Domain wall fermions and QCD}

Even if the domain wall fermion approach to chiral gauge theories on the lattice may fail, there could be a quite unexpected application of this idea. As put forward by Shamir [43, 71] domain wall fermions could be used for simulating QCD. This seems to be a natural approach since the odd dimensional theory one starts with is vectorlike. Consider a slightly modified action for domain wall fermions where we choose $2 N$ points in the $s$-direction.

$$
\begin{aligned}
S_{F} & =\sum_{x, s} \sum_{\mu=1}^{4} \bar{\psi}_{x}^{s}\left[(\not \partial(U)-\Delta(U)+m] \psi_{x}^{s}\right. \\
& +\frac{1}{2} \sum_{x, s} \bar{\psi}_{x}^{s}\left[\delta_{s, s+\mu_{5}}\left(1+\gamma_{5}\right)+\delta_{s-\mu_{5}, s}\left(1-\gamma_{5}\right)-2 \delta s, s\right] \psi_{x}^{s} \\
& +\bar{m} \bar{\psi}_{x}^{2 N}\left(1+\gamma_{5}\right) \psi_{x}^{1}+\bar{m} \psi_{x}^{1}\left(1-\gamma_{5}\right) \psi_{x}^{2 N} .
\end{aligned}
$$

This action is identical to eq.(110) except that the boundary term in the $s$-direction is made explicit. The gauge fields are kept 4-dimensional. The boundary terms are equipped with an additional new parameter $\bar{m}$. For $\bar{m}=1$, which corresponds to periodic boundary conditions, no zeromodes appear. For $\bar{m}=0$ we will have open boundary conditions and the action (141) describes boundary fermions with chiral surface modes 43.

For a non-zero value of $\bar{m}$ an interaction of the surface modes at $s=1$ and $s=2 N$ is induced and they will combine to a Dirac state. In order to derive the mass dependence of this state we compute the propagator from the action (141). First there is a homogeneous solution of the Dirac equation

$$
G_{0}\left(s, s^{\prime}\right)=B e^{-\alpha\left|s-s^{\prime}\right|}
$$

with

$$
\begin{aligned}
B^{-1}= & 2 b \sinh \alpha \\
2 \cosh \alpha(p)= & \frac{1+b^{2}(p)+\bar{p}^{2}}{b(p)} \\
\bar{p}=\sin p_{\mu} & b(p)=1-m+\sum_{\mu}\left(1-\cos \left(p_{\mu}\right)\right) .
\end{aligned}
$$

The full propagator is given as

$$
\begin{aligned}
G\left(s, s^{\prime}\right) & =G_{0}\left(s, s^{\prime}\right)+A_{-} e^{-\alpha\left(s+s^{\prime}\right)}+A_{+} e^{-\alpha\left(2 N-s-s^{\prime}\right)} \\
& +A_{m}\left(e^{-\alpha\left(N+s-s^{\prime}\right)}+e^{-\alpha\left(N+s^{\prime}-s\right)}\right)
\end{aligned}
$$


where

$$
\begin{aligned}
A_{-} & =\Delta^{-1} B b\left(1-\bar{m}^{2}\right)\left(e^{\alpha}-b\right) \\
A_{+} & =\Delta^{-1} B b\left(1-\bar{m}^{2}\right)\left(e^{-\alpha}-b\right) \\
A_{m} & =2 \Delta^{-1} B b^{2} \bar{m} \cosh \alpha \\
\Delta & =b e^{\alpha}-1+\bar{m}^{2} b\left(e^{\alpha}-b\right) .
\end{aligned}
$$

This complicated expression can be evaluated in the limit of small momenta and small values of $\bar{m}$. As a result one finds that the propagator in momentum space is

$$
G\left(p^{2}\right)=p^{2}+\bar{m}^{2} m^{2}(2-m)^{2} ; p^{2}, \bar{m}^{2} \ll 1 .
$$

For the boundary fermion model $\bar{m}=0$. We see that in the low momentum limit the propagator describes a massless particle. The $s$-dependence of the full propagator (144) shows that the chiral zeromodes appear as surface modes on the boundary of the lattice in the $s$-direction. This is the same result as we discussed in section 2 where the zeromodes were computed in the Hamiltonian language. They are depicted in figure $1 \mathrm{~b}$.

Turning on $\bar{m}$, these zeromodes are coupled through the links connecting the last and the first $s$-slice and acquire a -Dirac- mass. As can be read off from (146) this mass is proportional to $\bar{m}$ which might therefore be interpreted as an external quark mass. The reason, why this result makes boundary fermions interesting for QCD simulations is the following. According to the nogo theorems chiral symmetry is broken on the lattice. However, as weak coupling perturbation theory shows, chiral symmetry will be restored in the continuum limit of lattice QCD. To reach this limit a careful tuning of the bare parameters in the lattice QCD action is necessary to approach the phase transition. The tuning of the pion mass to zero as a function of the bare parameters is non-trivial and demanding. In particular, there are $O(1 / a)$ corrections to the renormalized quark mass.

Eq.(146) offers a nice alternative. It suggests that quark masses only get multiplicative renormalization and that one has therefore only to tune $\bar{m}$ to zero in order to reach the phase transition. In this context, $\bar{m}$ might be thought of as an external source. In particular, the phase transition point is expected to be $\bar{m}=0$. For a finite lattice in the $s$-direction there is an additional complication. Since the wavefunctions of both modes on the walls fall off exponentially, there will be always an overlap of both quark modes in the middle of the lattice. The value of the quark's wavefunction can be estimated to be $(1-m)^{s}$. Therefore the extension of the lattice in the extra dimension should be $N \approx-\log (m)$. Whether the advantage to make tuning trivial will merit the introduction of an extra dimension can only be answered in actual simulations.

For a finite system the overlap of the surface modes lead to some anomalous effects in the middle of the lattice. The currents are defined as

$$
j_{\mu}=\frac{1}{2}\left(\bar{\psi}_{x}^{s}\left(1+\gamma_{\mu}\right) U_{x, \mu} \psi_{x+\mu}^{s}-\bar{\psi}_{x+\mu}^{s}\left(1-\gamma_{\mu}\right) U_{x-\mu, \mu}^{\dagger} \psi_{x}^{s}\right)
$$


for $\mu=1, \ldots, 4$ and

$$
j_{5}=\left\{\begin{array}{cc}
\bar{\psi}_{x}^{s} P_{R} \psi_{x}^{s+1}-\bar{\psi}_{x}^{s+1} P_{L} \psi_{x}^{s} & 1 \leq s<2 N \\
\bar{\psi}_{x}^{2 N} P_{R} \psi_{x}^{1}-\bar{\psi}_{x}^{1} P_{L} \psi_{x}^{2 N} & s=2 N
\end{array} .\right.
$$

Since the 5-dimensional theory is vectorlike, it is anomaly free and the divergence equation is $\partial_{\mu} j_{\mu}=-\partial_{5} j_{5}, \mu=1, \ldots, 4$. Written out explicitly

$$
\sum_{\mu=1}^{4} \partial_{\mu} j_{\mu}=\left\{\begin{array}{cc}
-j_{5}^{s=1}-\bar{m} j_{5}^{s=2 N} & s=1 \\
-\partial_{5} j_{5}^{s} & 1<s<2 N \\
-j_{5}^{s=2 N-1}-\bar{m} j_{5}^{s=2 N} & s=2 N
\end{array} .\right.
$$

One can define an axial current as in section 3

$$
A_{\mu}=-\sum_{s=1}^{2 N} \operatorname{sign}\left(N-s+\frac{1}{2}\right) j_{\mu} .
$$

Then the divergence is

$$
\partial_{\mu} A_{\mu}=2 \bar{m} j_{5}^{2 N}+2 j_{5}^{N} .
$$

Whereas $j_{5}^{N}$ appears to be derived from a usual classical massterm, $j_{5}^{2 N}$ is an additional anomalous term stemming from the overlap of the surface modes. As is shown in [71] this term vanishes with $N \rightarrow \infty$ as

$$
\exp \left(-c_{0} / g_{0}^{2}\right) \exp (-\Lambda N)
$$

with $c_{0}$ a constant, $g_{0}$ the gauge coupling and $\Lambda$ the confinement length. From this formula it is seen that for every $g_{0}$ fixed, the anomalous term vanishes if the extent of the lattice in the extra dimension is sent to infinity. This suggests that one might try to follow the program of the previous section and work in the $N=\infty$ limit directly. Since in the application of the domain wall model to QCD the effective action on the boundary is real and therefore does not possess the phase ambiguity we found for the chiral gauge theory case, the program of Narayanan and Neuberger can now be performed without subtleties involved. The derivation of the overlap formula for the QCD case can be worked out following [29] closely and an explicit formula is found in [71]. The vanishing of the anomalous term in the $N \rightarrow \infty$ limit constitutes a proof that axial symmetry is restored at the QCD phase transition. In the derivation of this result basically no approximation is involved. This amounts to the statement that restoration of axial symmetry will also appear at strong coupling and is valid not only to every order in weak coupling perturbation theory [1] but holds also non-perturbatively.

\section{$9 \quad$ Summary and conclusions}

In this article we summarized the status of domain wall fermions invented to construct a chiral gauge theory on the lattice. In the continuum domain wall fermions are obtained as chiral 
zeromodes bound to a -soliton shaped- mass defect living in an extra odd dimension. The domain wall model can be taken over to the finite lattice by choosing the mass defect to be a step function. Through the necessary introduction of boundary conditions a second anti-wall is generated and the chiral zeromodes appear pairwise as left and righthanded fermions. They are bound to the wall and the anti-wall, respectively, and fall off exponentially with increasing distance from the walls. Alternatively one may choose free boundary conditions in the extra dimension in which case the chiral modes appear as surface modes.

The charge flow in the domain wall model is according to the picture developed in the continuum by Callan and Harvey. The anomaly appearing in the zeromode current along the wall is compensated by the divergence of the Chern-Simons current induced by the heavy fermions that live off the wall in the extra dimension. The Callan-Harvey analysis can be performed on the lattice confirming the continuum picture at least, as long as the gauge fields are taken to be smooth and external. The correct strength of the -covariant- anomaly can be directly measured on the finite lattice.

The crucial step for the realization of a chiral gauge theory on the lattice is the coupling to dynamical gauge fields. The fluctuations of the gauge field can become strong and the fear is that they will lead to an interaction of the two chiral zeromodes combining them to a Dirac particle. In the waveguide model only one of the two domain walls is gauged. Interpreting the extra dimension as a sophisticated flavour space, the gauge fields are kept strictly 4-dimensional and the same for all flavours in a region around the "wall"-flavour, i.e. the location of the wall. At the boundaries of this "waveguide region" gauge invariance is lost. This can be repaired by introducing scalar or Stückelberg fields at the boundary of the waveguide region. The coupling of the scalar fields and the fermions are of the Yukawa-form and will be equipped with a Yukawacoupling $y$. For $y=0$ one essentially obtains the model with free boundary conditions and new fermionic zeromodes appear which can interact with the domain wall zeromode through the gauge fields. The important and crucial question for the model to succeed is then whether the fermionic spectrum at the waveguide boundary can be made massive such that the modes decouple in the continuum limit and one is left with only the chiral zeromode on the gauged wall.

The result of the investigations in the domain wall model can be given, comparing fig.9 and fig.13. These figures show the phase diagrams that would have led to a possible construction of a chiral gauge theory with domain wall fermions (fig.9) and the much more realistic phase diagram 3 in fig. 13 which prohibit such a construction. The phase diagram in fig.13 was obtained by a large- $y$ expansion in combination with numerical simulation results.

The interesting part of the phase diagram in fig.9 is the $\mathrm{PMS}_{1}$ phase. In this phase the fermions at the boundary of the waveguide region would have become massive. There the

\footnotetext{
${ }^{3}$ In Fig.13 the $\mathrm{PMS}_{2}$ phase is left out. The existence of this phase was not investigated. But even if it would exist, a construction of a chiral gauge theory would not be possible because all modes including the one on the domain wall become heavy.
} 


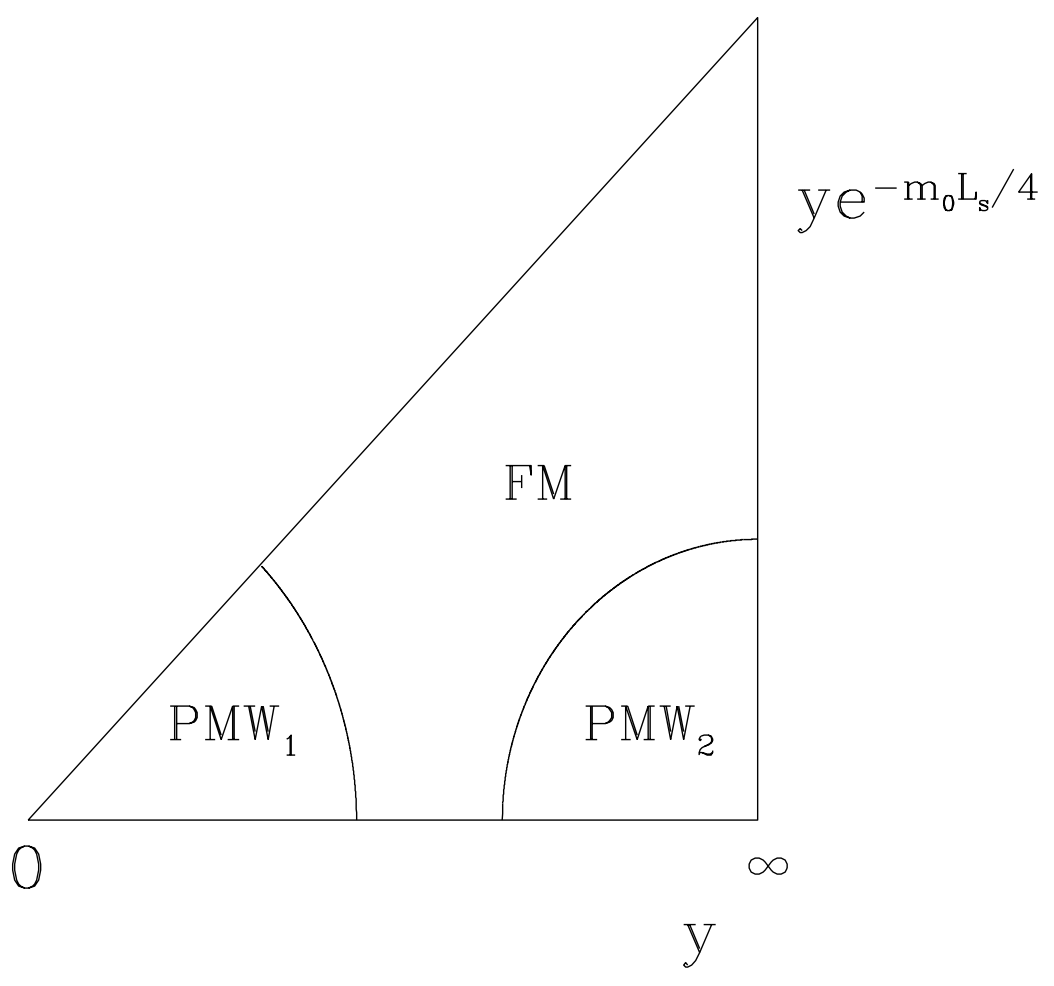

Figure 13: A realistic phase diagram for domain wall fermions. There will be a symmetry broken (FM) phase as well as two symmetric phases $\left(\mathrm{PMW}_{1}\right.$ and $\left.\mathrm{PMW}_{2}\right)$ with different vectorlike spectra of massless fermions.

Yukawa-coupling would have been strong enough to bind the fermion and the scalar field to form a massive bound state. Therefore this state would have decoupled from the low energy spectrum, leaving behind a chiral zeromode on a domain wall coupled to gauge fields in a gauge invariant manner. In earlier investigations of coupled fermion-Higgs models such a phase could be established or it was missing, depending on the particular implementation of the form of the Yukawa-coupling. What is found in the waveguide model from the numerical simulations and the large- $y$ expansion is that the $\mathrm{PMS}_{1}$ phase is absent. Instead, there are two symmetric phases with weak coupling behaviour both of them with a vectorlike spectrum of zeromodes. Thus in neither of these symmetric phases a chiral gauge theory can be obtained. A word of care has to be given at this point. Although the phase diagram in fig.13 represents a consistent picture from the large $y$ expansion and numerical simulations, the simulations were very difficult in the most interesting region of the phase diagram. Although evidences are strong that a $\mathrm{PMS}_{1}$ phase is absent, its non-existence could not be proven without any doubt. Maybe using the algorithm proposed in [65] can help to achieve better simulation results in the difficult region.

What remains from the domain wall model idea is a variant where the extra dimension is kept strictly infinite. In this interesting line of thinking the extra dimension is regarded as the time axis of a 4-dimensional Hamiltonian. The Hamiltonian is split into two parts corresponding to the two sides of the domain wall. Due to the infinite extension in the extra 
dimension the ground states on both sides of the wall are projected out. The resulting theory consists of the overlap of these two ground states. Assuming smooth external gauge fields it could be shown in perturbation theory and non-perturbatively through a diagonalization of the finite lattice Hamiltonians that this formulation of the domain wall model gives again the correct -consistent- anomalies. So far, in this interesting approach no dynamical gauge fields have been considered and it remains to be seen whether the promising results survive their inclusion.

A completely different application results from the interpretation of the wall zeromodes as massless quarks. The boundaries of the lattice in the extra dimension can be coupled through a mass term. In this way, the surface modes combine to build a Dirac fermion. The interesting aspect of this approach is that the mass of the Dirac-particle is directly proportional to the new mass parameter. Thinking of simulations in QCD this would mean that one could perhaps avoid the fine tuning problem of the quark mass in the conventional formulations of lattice QCD.

To conclude, the clash between chiral gauge invariance and the existence of a single Weyl fermion on the lattice could also not be resolved by the domain wall fermion approach. It seems that non-perturbative lattice models of chiral gauge theories unavoidingly lead to doubler states which might be interpreted as mirror fermions. One is therefore left with the choice to believe in some -yet undiscovered- new fermions, or to think further, maybe along the lines of the Rome-approach [72], how nature managed to construct chiral gauge theories.

\section{Acknowledgement}

This work would not have been possible without the very enjoyable collaboration with M.F.L. Golterman, D.B. Kaplan, D.N. Petcher, M. Schmaltz and J.C. Vink. I am grateful to them for numerous discussions and suggestions. In particular I would like to thank M.F.L. Golterman for reading the manusscript. 


\section{References}

[1] L.H. Karsten and J. Smit, Nucl. Phys. B183 (1981) 103.

[2] H. B. Nielsen and M. Ninomiya, Nucl. Phys. B185 (1981) 20; erratum: Nucl. Phys. B195 (1982) 541; Nucl. Phys. B193 (1981) 173; Phys. Lett. 105B (1981) 219.

[3] E. Eichten and J. Preskill, Nucl. Phys. B268 (1986) 179.

[4] J. Smit, Nucl. Phys. B (Proc. Suppl.) 17 (1990) 3.

[5] K.G. Wilson, in "New phenomena in subnuclear physics", ed. A. Zichichi (Plenum, New York, 1977) (Erice 1977).

[6] S.L. Adler, in "1970 Brandeis University Summer Institute in Theoretical Physics", ed. S. Deser, M. Grisaru and H. Pendleton (MIT Press, Cambridge, Mass.)

[7] J. Smit, Nucl. Phys. B (Proc. Suppl.) 4 (1988) 451.

[8] M.F.L. Golterman, Nucl. Phys. B (Proc. Suppl.) 20 (1991) 528.

[9] I. Montvay, Nucl. Phys. B (Proc. Suppl.) 26 (1992) 57.

[10] D.N. Petcher, Nucl. Phys. B (Proc. Suppl.) 30 (1993) 50.

[11] Nucl. Phys.B (Proc.Suppl.) 29B,C (1992).

[12] H.B. Nielsen and S.E. Rugh, Nucl. Phys.B (Proc.Suppl.) 29B,C (1992) 200.

[13] D.B. Kaplan, Phys. Lett B288 (1992) 342.

[14] R. Jackiw and C. Rebbi, Phys.Rev.D13 (1976) 3398.

[15] C.G. Callan Jr. and J.A. Harvey, Nucl. Phys. B250 (1985) 427.

[16] P.H. Frampton and T.W. Kephart, Phys.Rev. 50 (1983) 1343,1347; Phys.Rev.D28 (1983) 1010; L. Alvarez-Gaumé and E. Witten, Nucl.Phys.B234 (1983) 269; B. Zumino, Lectures at Les Houches Summer School (1983); R. Stora Cargése Lectures (1983).

[17] J. Goldstone and F. Wilczek, Phys.Rev.Lett 47 (1981) 986.

[18] D. Boyanovsky, E. Dagotto and E. Fradkin, Nucl. Phys. B285 (1987) 340; E. Fradkin, Nucl.Phys. B (Proc.Suppl.)1A (1987) 175; E. Dagotto, E. Fradkin and A. Moreo, Phys.Lett. 172B (1986) 383.

[19] S. Aoki and H. Hirose, Phys.Rev.D49 (1994) 2604.

[20] K. Jansen, Phys. Lett. B288 (1992) 348. 
[21] K. Jansen and M. Schmaltz, Phys. Lett. B 296 (1992) 374.

[22] M.F.L. Golterman, K. Jansen and D.B. Kaplan, Phys. Lett. B301 (1993) 219.

[23] Z. Yang, Phys.Lett.B296 (1992) 151.

[24] C.P. Korthals-Altes, S. Nicolis and J. Prades, Phys. Lett. B316 (1993) 339.

[25] M.F.L. Golterman, K. Jansen, D.N. Petcher and J. Vink, Phys.Rev.D49 (1994) 1606.

[26] T. Kawano and Y. Kikukawa, "On the large mass limit of Continuum theories in Kaplan's Formulation", Kyoto Univ. preprint, KUNS-1239.

[27] H. Aoki, S. Ito, J. Nishimura and M. Oshikawa, "The effect of dynamical gauge field on the chiral fermion on a boundary", KEK preprint, KEK-TH-393.

[28] D. Kaplan, Nucl.Phys. B (Proc.Suppl.) 30 (1993) 597.

[29] R. Narayanan and H. Neuberger, Phys. Lett. B302 (1993)62; Phys.Rev.Lett.71 (1993) 3251; Nucl.Phys.B412 (1994) 574; Nucl.Phys.B (Proc. Suppl.) 34 (1994) 587.

[30] A.K. De and J. Jersák, "Yukawa models on the lattice", HLRZ Jülich preprint, HLRZ 91-83.

[31] J. Shigemitsu, "Yukawa coupling generated fermion masses for Wilson lattice fermions", Ohio State University preprint, DOE/ER/01545-397.

[32] A. Hasenfratz and T. Neuhaus, Phys.Lett.B220 (1989) 435.

[33] W. Bock, A.K. De, K. Jansen, J. Jersák, T. Neuhaus and J. Smit, Nucl. Phys. B344 (1990) 207.

[34] J. Distler and S.-J. Rey, "3 into 2 doesn't go", Princeton preprint, PUPT-1386.

[35] J. Smit, Acta Physica Polonica B17 (1986) 531 (Zakopane 1985).

[36] I. Montvay and G. Münster, "Quantum Fields on a Lattice", Cambridge University Press, 1994.

[37] M. Bochicchio, L. Maiani, G. Martinelli, G.C. Rossi and M. Testa, Nucl.Phys.B262 (1985) 331; C. Curci, Phys.Lett.B167 (1986) 425.

[38] P.D.V. Swift, Phys.Lett.B 145 (1984) 256.

[39] S. Hands and D.B. Carpenter, Nucl.Phys.B266 (1986) 285.

[40] W. Bock, A.K. De, C. Frick, K. Jansen and T. Trappenberg, Nucl.Phys. B371 (1992) 683. 
[41] M.F.L. Golterman, D.N. Petcher and J. Smit, Nucl.Phys. B370 (1992) 51.

[42] W. Bock, A.K. De and J. Smit, Nucl.Phys. B388 (1992) 243.

[43] Y. Shamir, Nucl.Phys. B406 (1993) 90.

[44] M. Creutz and I. Horvath, "Surface states and chiral symmetry on the lattice", BNLpreprint, BNL-60062.

[45] Y. Shamir, Nucl.Phys. B417 (1993) 167.

[46] S. Chandrasekharan, Phys.Rev.D49 (1994) 1980.

[47] A. Coste and M. Lüscher, Nucl.Phys.B323 (1989) 631.

[48] H. So, Prog.Theor.Phys. 73 (1985) 528; 74 (1985) 585.

[49] S.G. Naculich, Nucl.Phys.B296 (1988) 837.

[50] J. Wess and B. Zumino, Phys.Lett. B37 (1971) 95.

[51] W.A. Bardeen and B. Zumino, Nucl.Phys.B244 (1984) 421.

[52] Y. Shamir, Phys.Lett.B305 (1992) 357.

[53] T. Banks and A. Dabholkar, Phys.Rev.D46 (1992) 4016; M.J. Dugan and A.V. Manohar, Phys.Lett.B265 (1991) 137; W. Bock, J.E. Hetrick and J. Smit, UCSD preprint, "Fermion production despite fermion number conservation", UCSD/PTH 94-07.

[54] K. Jansen, Nucl.Phys. B (Proc.Suppl.) 30 (1993) 601.

[55] R. Balian, J.M. Drouffe and C. Itzykson, Phys.Rev.D11 (1975) 2098;

J.M. Drouffe and J.-B. Zuber, Phys.Rep. 102 (1983) 1.

[56] Y.K. Fu and H.B. Nielsen, Nucl.Phys.B236 (1984) 167.

[57] G. Parisi, Nucl.Phys.B (Proc.Suppl.) 29B,C (1992) 247.

[58] I. Montvay, Phys. Lett. B199 (1987) 89; Nucl. Phys. B (Proc. Suppl.) 29B,C (1992) 159.

[59] A.K. De, E. Focht, W. Franzki, J. Jersák and M.A. Stephanov, Phys. Lett. B308 (1993) 32.

[60] M.F.L. Golterman and Y. Shamir, "Domain wall fermions in a waveguide: the phase diagram at large Yukawa coupling", Washington University preprint, Wash.U.HEP/94-61.

[61] I-H. Lee, J. Shigemitsu and R.E. Shrock, Nucl. Phys. B330 (1990) 225. 
[62] A.Hasenfratz, K. Jansen, C.B. Lang, T. Neuhaus and H. Yoneyama, Phys.Lett.B199 (1987) 531; J. Kuti, L.Lin and Y. Shen, Phys.Rev.Lett.61 (1988) 678; A. Hasenfratz, K. Jansen, J. Jersák, H.A. Kastrup, C.B. Lang, H. Leutwyler and T. Neuhaus, Nucl.Phys.B356 (1991) 332.

[63] I. Barbour, W. Bock, C. Davies, A.K. De, D. Hently, J. Smit and T. Trappenberg, Nucl. Phys. B368 (1992) 390.

[64] S. Duane, A.D. Kennedy, B.J. Pendleton and D. Roweth, Phys.Lett.B195 (1987) 216.

[65] M. Lüscher, Nucl.Phys.B418 (1994) 637.

[66] R. Narayanan, Nucl.Phys.B (Proc.Suppl.) 34 (1994) 95.

[67] S.A. Frolov and A.A. Slavnov, Nucl.Phys.B411 (1994) 647.

[68] M. Lüscher, Commun.Math.Phys. 54 (1977) 283.

[69] L. Alvarez-Gaumé, S. Della Pietra and V. Diella Pietra, Phys.Lett.B166 (1986) 177.

[70] M. Göckeler and G. Schierholz, Nucl.Phys.B (Proc.Suppl.) 30 (1993) 609; Nucl. Phys.B (Proc.Suppl.) 29B,C (1992).

[71] V. Furman and Y. Shamir, "Axial Symmetries in lattice QCD with Kaplan fermions", Weizmann Institute preprint, WIS-94/19.

[72] A. Borelli, L. Maiani, G.C. Rossi, R. Sisto and M. Testa, Phys.Lett. B221 (1989) 360; Nucl.Phys.B333 (1990) 355; L. Maiani, Nucl.PhysB (Proc. Suppl.) 29B,C (1992) 33; J.C. Vink, Phys.Lett.B321 (1994) 239. 